\title{
Spectral flow of chiral fermions in nondissipative Yang-Mills gauge field backgrounds
}

\author{
F.R. Klinkhamer* and Y.J. Lee ${ }^{\dagger}$ \\ Institut für Theoretische Physik, Universität Karlsruhe, D-76128 Karlsruhe, Germany \\ (preprint KA-TP-09-2001, hep-th/0104096; Physical Review D 64, 065024 (2001))
}

\begin{abstract}
Real-time anomalous fermion number violation is investigated for massless chiral fermions in spherically symmetric $S U(2)$ Yang-Mills gauge field backgrounds which can be weakly dissipative or even nondissipative. Restricting consideration to spherically symmetric fermion fields, the zero-eigenvalue equation of the time-dependent effective Dirac Hamiltonian is studied in detail. For generic spherically symmetric $S U(2)$ gauge fields in Minkowski spacetime, a relation is presented between the spectral flow and two characteristics of the background gauge field. These characteristics are the well-known "winding factor," which is defined to be the change of the Chern-Simons number of the associated vacuum sector of the background gauge field, and a new "twist factor," which can be obtained from the zero-eigenvalue equation of the effective Dirac Hamiltonian but is entirely determined by the background gauge field. For a particular class of (weakly dissipative) Lüscher-Schechter gauge field solutions, the level crossings are calculated directly and nontrivial contributions to the spectral flow from both the winding factor and the twist factor are observed. The general result for the spectral flow may be relevant to electroweak baryon number violation in the early universe.
\end{abstract}

PACS numbers 11.15.-q, 03.65.Pm, 11.30.Fs, 11.30.Rd

Typeset using REVTEX

*E-mail: frans.klinkhamer@physik.uni-karlsruhe.de

†E-mail:lee@particle.physik.uni-karlsruhe.de 


\section{INTRODUCTION}

An important consequence of the triangle anomaly [1,2] is the violation of fermion number conservation in the electroweak Standard Model if the background gauge field has nonvanishing topological charge $Q$. This connection between the triangle anomaly and fermion number violation in the electroweak Standard Model was first pointed out in Ref. [3]. For the background gauge field, the calculation of Ref. [3] used Euclidean instanton solutions [4] in order to calculate the tunneling amplitude between topologically different vacua. A special feature of the Euclidean (imaginary-time) approach is that gauge field configurations with finite action fall into homotopy classes labeled by an integer $Q$. This integer $Q$ then gives the number of fermions produced.

For real-time fermion-number-violating processes [5], sphaleron-like gauge field configurations are believed to play crucial role [6]. These gauge fields "interpolate" between topologically different vacua and have sufficiently high energy to overcome the energy barrier. But, in contrast with the Euclidean approach, the anomalous fermion production in Minkowski spacetime is not, in general, given by the topological charge $Q$ of the classical gauge field background. The reason is that $Q$ may be a noninteger or even, for the case of Yang-Mills-Higgs theory, not well-defined; cf. Refs. [7,8]. It is not clear which quantity, in general, determines the anomalous fermion production for real-time processes.

For pure Yang-Mills theory in Minkowski spacetime, the authors of Refs. [9, 10] have argued that the number of produced fermions is given by the change of winding number of the associated vacua of the initial and final gauge field configurations. The "associated vacua" of a given classical gauge field background represent the particular vacuum configurations that the background field would approach as $t \rightarrow-\infty$ or $t \rightarrow+\infty$. As is clear from the context, the authors of Refs. [9, 10] considered dissipative background fields in order to be able to quantize the fermion fields at $t= \pm \infty$.

The question, now, is what happens to fermion number violation if a powerful energy source creates a nontrivial gauge field background over an extended spacetime region (for example, in a high-energy collision experiment or in the early universe). In this case, we cannot readily associate the initial high-energy state with a particular vacuum configuration and the field-theoretic approach used in Refs. [9, 10] breaks down. For nontrivial classical bosonic background fields, it is, moreover, not known how to construct a fermion number operator in terms of the quantized fermionic fields .

Still, fermion number violation can be directly observed from the level crossing of the energy eigenvalues of the time-dependent Dirac Hamiltonian, see Ref. [5] and references therein. The overall effect of level crossing can be characterized by the "spectral flow," defined to be the number of eigenvalues of the Dirac Hamiltonian that cross zero from below minus the number of eigenvalues that cross zero from above, for a given time interval and direction of time.

In this paper, we study the zero-eigenvalue equation of the effective Dirac Hamiltonian for spherically symmetric chiral fermion fields and classical $S U(2)$ Yang-Mills gauge field backgrounds. A relation is found between the spectral flow and certain features of the spherically symmetric background gauge field. These features are the well-known topological "winding factor" and a new type of "twist factor," both of which will be defined later. The spherically symmetric subspace of $(3+1)$-dimensional chiral $S U(2)$ Yang-Mills theory 
is equivalent to an $(1+1)$-dimensional $U(1)$ gauge theory coupled to a Higgs-like complex scalar field [11] and several two-component Dirac fields. This drastic simplification allows us to examine the problem using analytical methods.

The (3+1)-dimensional spherically symmetric $S U(2)$ gauge field backgrounds considered in this paper are, in general, nondissipative, which means that the energy density does not approach zero uniformly as $t \rightarrow \pm \infty$. For these nondissipative gauge field backgrounds, the contribution of the twist factor to the spectral flow is manifest. The general result for the spectral flow also applies to dissipative spherically symmetric gauge field backgrounds, which will be classified later as "weakly dissipative" and "strongly dissipative." It will be shown that the nonvanishing effect of the twist factor for the spectral flow can already appear for weakly dissipative spherically symmetric gauge field backgrounds, such as certain Lüscher-Schechter gauge field solutions [12, 13]. Our paper may, therefore, be viewed as a continuation of the work of Refs. [5, 9, 10].

The outline of this paper is as follows. In Section II, we present the model and the basic formalism. After giving the chiral $S U(2)$ Yang-Mills theory in the spherically symmetric Ansatz, we briefly review the topological properties of the gauge field background. The gauge field topology is, in the first place, characterized by the winding factor, defined to be the change of the Chern-Simons number of the associated vacuum sector of the gauge field configuration.

In Section III, we consider the zero-eigenvalue equation of the time-dependent effective Dirac Hamiltonian. By investigating the zero-eigenvalue equation directly, we are able to identify a family of Riccati equations [14,15], from which the twist factor of the spherically symmetric $S U(2)$ gauge field configuration can be obtained.

In Section IV, we present a result for generic spherically symmetric $S U(2)$ gauge field backgrounds, which relates the spectral flow to both the winding factor and the twist factor.

In Section V, we investigate the level crossing phenomenon for the particular spherically symmetric $S U(2)$ gauge field backgrounds given by certain Lüscher-Schechter solutions 12.13 and verify our relation for the spectral flow. Specifically, we demonstrate the significant effect of the twist factor for a class of Lüscher-Schechter background gauge fields with energies far above a sphaleron-like barrier.

In Section VI, finally, we summarize our results and briefly discuss the role of dissipation. There is also an appendix, which provides the proof of a result needed in this section.

For the benefit of the reader, we remark that Sections II B, III C, and IV B form the core of the paper.

\section{CHIRAL $S U(2)$ YANG-MILLS THEORY}

In this section, we review the spherically symmetric Ansatz for massless chiral fermions coupled to classical $S U(2)$ Yang-Mills gauge fields and establish our notation. Furthermore, we recall the definition of the topological winding factor.

\section{A. Spherically symmetric Ansatz}

The $S U(2)$ Yang-Mills theory with massless chiral fermions is described by the action, 


$$
S=S_{G}+S_{F}, \quad S_{G}=-\frac{1}{2 g^{2}} \int_{\mathbb{R}^{4}} \mathrm{~d}^{4} x \operatorname{Tr}\left(F^{m n} F_{m n}\right), \quad S_{F}=\int_{\mathbb{R}^{4}} \mathrm{~d}^{4} x \bar{\Psi}_{f} \Gamma^{m} D_{m} \Psi_{f},
$$

where $S_{G}$ represents the gauge field action and $S_{F}$ the fermionic action. Latin indices $m$, $n$, etc. run over the coordinate labels $0,1,2,3$, and the metric tensor for flat Minkowski spacetime is $\eta_{m n}=\operatorname{diag}(-1,1,1,1)$. Repeated indices are summed over. The flavor index $f$, in particular, is summed over $1, \ldots, N_{F}$. Also, natural units are used for which $c=\hbar=$ 1.

The $S U(2)$ field strength tensor $F_{m n}$ and the covariant derivative $D_{m}$ for the fermionic fields are defined as follows:

$$
\begin{aligned}
F_{m n} & \equiv \partial_{m} A_{n}-\partial_{n} A_{m}+\left[A_{m}, A_{n}\right], \quad A_{m} \equiv A_{m}^{a} \tau^{a} /(2 i) \\
D_{m} & \equiv \partial_{m}+A_{m} \mathrm{P}_{L}, \quad \mathrm{P}_{L} \equiv\left(1-\Gamma_{5}\right) / 2, \quad \mathrm{P}_{R} \equiv\left(1+\Gamma_{5}\right) / 2
\end{aligned}
$$

The Dirac matrices $\Gamma^{m}$ are taken in the chiral (Weyl) representation,

$$
\begin{aligned}
& \Gamma^{0}=-i\left(\begin{array}{ll}
0 & \mathbb{1} \\
\mathbb{1} & 0
\end{array}\right), \quad \Gamma^{a}=-i\left(\begin{array}{cc}
0 & \sigma^{a} \\
-\sigma^{a} & 0
\end{array}\right), \quad \Gamma_{5} \equiv-i \Gamma^{0} \Gamma^{1} \Gamma^{2} \Gamma^{3}=\left(\begin{array}{cc}
\mathbb{1} & 0 \\
0 & -\mathbb{1}
\end{array}\right), \\
& \mathbb{1} \equiv\left(\begin{array}{ll}
1 & 0 \\
0 & 1
\end{array}\right), \quad \sigma^{1} \equiv\left(\begin{array}{ll}
0 & 1 \\
1 & 0
\end{array}\right), \quad \sigma^{2} \equiv\left(\begin{array}{cc}
0 & -i \\
i & 0
\end{array}\right), \quad \sigma^{3} \equiv\left(\begin{array}{cc}
1 & 0 \\
0 & -1
\end{array}\right) .
\end{aligned}
$$

The conjugate spinor is given by $\bar{\Psi}_{f} \equiv \Psi_{f}^{\dagger}\left(-i \Gamma^{0}\right)$. Here and in the following, $\tau^{a}$ and $\sigma^{a}$ are Pauli matrices carrying isospin and spin indices, respectively. The action (2.1) thus corresponds to a chiral $S U(2)$ gauge theory, with interacting left-handed fermions $\left(\Psi_{L f} \equiv\right.$ $\left.P_{L} \Psi_{f}\right)$ and noninteracting right-handed fermions $\left(\Psi_{R f} \equiv P_{R} \Psi_{f}\right)$.

The total number $N_{F}$ of flavors in the fermionic action (2.1) must be even, in order to cancel the nonperturbative $S U(2)$ anomaly [16]. Henceforth, we focus on a single flavor and drop the index $f$. Since there is no natural mass scale for the classical $S U(2)$ Yang-Mills theory, we also take an arbitrary mass scale to work with. (In the full theory, quantum effects may, of course, fix the scale.)

In this paper, we concentrate on the spherically symmetric subspace of the $(3+1)$ dimensional theory. We use the following Ansatz for the gauge fields:

$$
\begin{aligned}
& A_{0}(x)=\frac{1}{2 i} a_{0}(t, r) \vec{\tau} \cdot \hat{\mathbf{x}} \\
& A_{a}(x)=\frac{1}{2 i}\left[\frac{\alpha(t, r)-1}{r} \epsilon_{a b c} \hat{x}_{c} \tau_{b}+\frac{\beta(t, r)}{r}\left(\delta_{a b}-\hat{x}_{a} \hat{x}_{b}\right) \tau_{b}+a_{1}(t, r)(\vec{\tau} \cdot \hat{\mathbf{x}}) \hat{x}_{a}\right]
\end{aligned}
$$

where $\alpha, \beta, a_{0}$, and $a_{1}$ are real functions of $t$ and $r$; cf. Refs. [11,17. These gauge fields are invariant under spatial $S O(3)$ rotations, up to a gauge transformation

$$
A_{m} \rightarrow A_{m}^{\Omega} \equiv \Omega\left(\partial_{m}+A_{m}\right) \Omega^{\dagger},
$$

with $\Omega(x) \in S U(2)$.

The spherically symmetric Ansatz for the fermionic fields is given by (see Ref. [17] and references therein) 


$$
\begin{gathered}
\Psi(x)=\left(\begin{array}{c}
\Psi_{R}(x) \\
\Psi_{L}(x)
\end{array}\right) \equiv\left(\begin{array}{c}
\tau^{2} \tilde{\Psi}_{R}(x) \\
\tilde{\Psi}_{L}(x)
\end{array}\right), \\
\tilde{\Psi}_{L}(x)=\frac{1}{\sqrt{2}}\left[H_{L}(t, r)+i G_{L}(t, r) \vec{\tau} \cdot \hat{\mathbf{x}}\right]\left(\begin{array}{c}
\left(\begin{array}{c}
0 \\
+1
\end{array}\right)_{\text {isospin }} \\
\left(\begin{array}{c}
-1 \\
0
\end{array}\right)_{\text {isospin }}
\end{array}\right)_{\text {spin }}, \\
\tilde{\Psi}_{R}(x)=\frac{1}{\sqrt{2}}\left[H_{R}(t, r)+i G_{R}(t, r) \vec{\tau}^{*} \cdot \hat{\mathbf{x}}\right]\left(\begin{array}{c}
+1 \\
0 \\
0 \\
0 \\
\left(\begin{array}{c}
+1
\end{array}\right)_{\text {isospin }}
\end{array}\right)_{\text {spin }},
\end{gathered}
$$

where $H_{L}, H_{R}, G_{L}$, and $G_{R}$ are complex functions of $t$ and $r$. In components ( $a$ for isospin and $\alpha$ for spin), the two constant spinors of Eqs. (2.6 b,c) can be written as $\epsilon_{a \alpha}$ and $\delta_{a \alpha}$, respectively, where $\epsilon$ and $\delta$ are the Levi-Civita and Kronecker symbols.

Furthermore, we assume that all physical $(3+1)$-dimensional field configurations are described by infinitely differentiable functions (this assumption can be relaxed). In order to have regular behavior at the spatial origin for the $(3+1)$-dimensional field configurations and their derivatives, the Ansatz functions should satisfy the following $r$-parity expansions near $r=0$ :

$$
\begin{aligned}
a_{0}(t, r) & =\sum_{k=0}^{\infty} a_{0}^{(2 k+1)}(t) r^{2 k+1}, & a_{1}(t, r) & =\sum_{k=0}^{\infty} a_{1}^{(2 k)}(t) r^{2 k}, \\
\alpha(t, r) & =1+\sum_{k=1}^{\infty} \alpha^{(2 k)}(t) r^{2 k}, & \beta(t, r) & =\sum_{k=0}^{\infty} \beta^{(2 k+1)}(t) r^{2 k+1}, \quad \beta^{(1)}(t)=a_{1}^{(0)}(t), \\
H_{L, R}(t, r) & =\sum_{k=0}^{\infty} H_{L, R}^{(2 k)}(t) r^{2 k}, & G_{L, R}(t, r) & =\sum_{k=0}^{\infty} G_{L, R}^{(2 k+1)}(t) r^{2 k+1},
\end{aligned}
$$

with the expansion coefficients depending on time only.

If we substitute the Ansätze (2.4) and (2.6) into the action (2.1), the following reduced actions are obtained (for a single fermion flavor):

$$
\begin{aligned}
& S_{G}=\frac{4 \pi}{g^{2}} \int_{-\infty}^{+\infty} \mathrm{d} t \int_{0}^{\infty} \mathrm{d} r\left\{\frac{1}{4} r^{2} f_{\mu \nu} f^{\mu \nu}+\left|D_{\mu} \chi\right|^{2}+\frac{1}{2 r^{2}}\left(|\chi|^{2}-1\right)^{2}\right\}, \\
& S_{F}=4 \pi \int_{-\infty}^{+\infty} \mathrm{d} t \int_{0}^{\infty} \mathrm{d} r\left\{\bar{\Psi}_{l}\left(\gamma^{\mu} D_{\mu}+\frac{1}{r}\left(\operatorname{Re} \chi+i \gamma_{5} \operatorname{Im} \chi\right)\right) \Psi_{l}+\bar{\Psi}_{r}\left(\gamma^{\mu} \partial_{\mu}+\frac{1}{r}\right) \Psi_{r}\right\} .
\end{aligned}
$$

Greek indices $\mu, \nu$, etc. run over the coordinate labels 0,1 , and are lowered with the metric $\eta_{\mu \nu} \equiv \operatorname{diag}(-1,1)$. The coordinates $\left(x^{0}, x^{1}\right)$ correspond to $(t, r)$. The theory (2.8) can be interpreted as an $(1+1)$-dimensional $U(1)$ gauge field theory with a Higgs-like complex scalar field $\chi(t, r)$ and two-component Dirac spinors $\Psi_{l}(t, r)$ and $\Psi_{r}(t, r)$. In terms of the Ansatz functions, the $U(1)$ field strength $f_{\mu \nu}$, the complex scalar and Dirac fields, and the covariant derivatives are given by:

$$
f_{\mu \nu} \equiv \partial_{\mu} a_{\nu}-\partial_{\nu} a_{\mu}, \quad \chi \equiv \alpha+i \beta, \quad D_{\mu} \chi \equiv\left(\partial_{\mu}-i a_{\mu}\right) \chi,
$$




$$
\begin{aligned}
\Psi_{l}(t, r) & \equiv\left(\begin{array}{c}
\Psi_{l 1}(t, r) \\
\Psi_{l 2}(t, r)
\end{array}\right) \equiv\left(\begin{array}{c}
r H_{L}(t, r) \\
r G_{L}(t, r)
\end{array}\right), \quad \Psi_{r}(t, r) \equiv\left(\begin{array}{c}
r H_{R}(t, r) \\
r G_{R}(t, r)
\end{array}\right), \\
\bar{\Psi}_{l, r} & \equiv \Psi_{l, r}^{\dagger}\left(-i \gamma^{0}\right), \quad D_{\mu} \Psi_{l} \equiv\left(\partial_{\mu}+i\left(a_{\mu} / 2\right) \gamma_{5}\right) \Psi_{l},
\end{aligned}
$$

with

$$
\gamma^{0}=i \sigma^{1}, \quad \gamma^{1}=-\sigma^{3}, \quad \gamma_{5}=-\gamma^{0} \gamma^{1}=\sigma^{2}
$$

The spherically symmetric Ansatz (2.4), (2.6) preserves a $U(1)$ subgroup of the $S U(2)$ gauge group, with transformation parameters $\Omega(\mathbf{x}, t)=\exp [i \omega(t, r) \tau \cdot \hat{\mathbf{x}} / 2]$ in Eq. (2.5) above. Under these particular $S U(2)$ gauge transformations, we have for the $(1+1)$ dimensional fields the following $U(1)$ gauge transformations:

$$
a_{\mu} \rightarrow a_{\mu}+\partial_{\mu} \omega, \quad \chi \rightarrow e^{i \omega} \chi, \quad \Psi_{l} \rightarrow e^{-i(\omega / 2) \gamma_{5}} \Psi_{l}, \quad \Psi_{r} \rightarrow \Psi_{r}
$$

In order to maintain the regularity of the $(3+1)$-dimensional field configurations, $\omega(t, r)$ should have an odd $r$-parity expansion near $r=0$,

$$
\omega(t, r)=\sum_{k=0}^{\infty} \omega^{(2 k+1)}(t) r^{2 k+1},
$$

where the expansion coefficients are dependent only on time.

For later reference, the $(1+1)$-dimensional fields with finite energy approach a vacuum configuration at infinity, provided

$$
\chi \rightarrow e^{i \omega}, \quad D_{\mu} \chi \rightarrow 0, \quad a_{\mu} \rightarrow \partial_{\mu} \omega, \quad f_{\mu \nu} \rightarrow 0, \quad \Psi_{l, r} \rightarrow 0, \quad \text { for } \quad r \rightarrow \infty,
$$

and are regular at the spatial origin, provided

$$
|\chi| \rightarrow 1, \quad D_{\mu} \chi \rightarrow 0, \quad \Psi_{l, r} \rightarrow 0, \quad \text { for } \quad r \rightarrow 0 \text {. }
$$

See Ref. [17 for further details. Throughout this paper, we consider regular spherically symmetric $S U(2)$ gauge fields with finite energy.

\section{B. Gauge field winding factor}

For the description of the topology of spherically symmetric $S U(2)$ gauge field backgrounds, it is convenient to express the $(1+1)$-dimensional complex field $\chi(t, r)$ in polar form:

$$
\chi(t, r)=\rho(t, r) \exp [i \varphi(t, r)], \quad \rho(t, r) \geq 0 .
$$

The "associated vacuum sector" of the background gauge field at a fixed time $t$ is obtained from the configuration with $\rho(t, r)$ replaced by 1 , but with $\varphi(t, r)$ and $a_{\mu}(t, r)$ unchanged. [Note that the resulting configuration with $\rho(t, r)=1$ may still have nonzero energy density (2.8a).] For the gauge choice $\chi(t, 0)=\chi(t, \infty)=1$, the integer winding number is then defined as 


$$
N_{\chi}(t) \equiv[\varphi(t, \infty)-\varphi(t, 0)] /(2 \pi) .
$$

This winding number $N_{\chi}(t)$ is, in fact, equal to the Chern-Simons number of the $\rho=1$ gauge field at time $t$; see Eq. (2.18) of Ref. [17]. For a particular $\chi(t, r)$ configuration, the winding number $N_{\chi}(t)$ is unambiguous, provided $|\chi(t, r)|>0$. See Sec. IV B for further discussion.

For a time interval $\left[t_{i}, t_{f}\right]$ with $t_{i}<t_{f}$, generic spherically symmetric $S U(2)$ gauge field backgrounds are characterized by the change of winding number $N_{\chi}$ between the initial and final configurations,

$$
\Delta N_{\chi}\left[t_{f}, t_{i}\right] \equiv N_{\chi}\left(t_{f}\right)-N_{\chi}\left(t_{i}\right) .
$$

Henceforth, we call $\Delta N_{\chi}\left[t_{f}, t_{i}\right]$ as defined by Eq. (2.17) the "winding factor" of the spherically symmetric $S U(2)$ gauge field. Our definition of the "winding factor" is directly inspired by the results of Ref. [10], obtained for a particular class of background fields that will be discussed further in Sec. V.

\section{TIME-DEPENDENT DIRAC HAMILTONIAN AND TWIST FACTOR}

In this section, we consider the zero-eigenvalue equation of the time-dependent effective Dirac Hamiltonian for a given spherically symmetric $S U(2)$ gauge field configuration at one particular time. The existence of fermion zero modes is discussed and a necessary condition derived. In addition, the so-called twist factor is introduced, which will play an important role in Sec. IV.

\section{A. Fermion zero modes and level crossings}

The general solution $\Psi_{l}(t, r)$ of the $(1+1)$-dimensional Dirac equation from the action (2.8b) can be expressed as a linear combination of the eigenfunctions of the corresponding time-dependent Dirac Hamiltonian. The eigenvalue equation of this Hamilton operator is

$$
\begin{aligned}
H(t, r) \Psi(t, r)= & E(t) \Psi(t, r) \\
H(t, r) \equiv & \gamma_{5} a_{0} / 2-i \gamma_{5} D_{1} \\
& +i \gamma^{0}\left(\operatorname{Re} \chi+i \operatorname{Im} \chi \gamma_{5}\right) / r
\end{aligned}
$$

where the covariant derivative $D_{1}$ has been defined in Eq. (2.9) and $\Psi$ now stands for the two-component Dirac spinor $\Psi_{l}$ of that same equation. [The other Dirac field $\Psi_{r}(t, r)$ of the action (2.8b) has no interactions and will not be considered in the following.] The Hamiltonian (3.1b) depends on $t$ and $r$ through the background fields $\chi(t, r)$ and $a_{\mu}(t, r)$, together with an explicit dependence on $r$ in the $i \gamma^{0}$ term.

It is known that the zero-crossing of an energy eigenvalue of the Dirac Hamiltonian is one of the crucial ingredients of fermion number violation; cf. Refs. [5, 18]. In our case, the zero-eigenvalue equation (3.1a) at fixed time $t$ can be written as 


$$
\begin{aligned}
\partial_{r} \Psi & =\mathcal{A} \Psi, \quad \mathcal{A} \equiv \mathcal{A}_{H}+\mathcal{A}_{A} \\
\mathcal{A}_{H} & \equiv-\left(\gamma^{1} \operatorname{Re} \chi+i \gamma^{0} \operatorname{Im} \chi\right) / r \\
\mathcal{A}_{A} & \equiv-i a_{0} / 2-i \gamma_{5} a_{1} / 2
\end{aligned}
$$

where $\partial_{r}$ stands for the partial derivative with respect to $r$. For later convenience, we have decomposed the matrix $\mathcal{A}$ of Eq. (3.2a) into a Hermitian part $\mathcal{A}_{H}$ and an anti-Hermitian part $\mathcal{A}_{A}$. Recall that we use two-dimensional Dirac matrices $\gamma^{0}=i \sigma^{1}, \gamma^{1}=-\sigma^{3}$, and $\gamma_{5}=\sigma^{2}$, with $\sigma^{a}$ the standard $2 \times 2$ Pauli matrices.

In order to have a regular $(3+1)$-dimensional fermionic field at $r=0$, the $(1+1)$ dimensional fermionic field $\Psi(t, r)$ must satisfy the boundary condition $\Psi(t, 0)=0$, which is already implemented by the Ansatz (2.9). A fermion zero mode is then defined to be a normalizable solution of Eq. (3.2a) with boundary condition $\Psi(t, 0)=0$. Specifically, the normalization condition is given by

$$
\int_{0}^{\infty} \mathrm{d} r|\Psi(t, r)|^{2}=1
$$

The existence of a fermion zero mode at a particular time $t=t^{*}$ does not necessarily imply level crossing of the eigenvalue of the Dirac Hamilton operator. In fact, the energy level $E(t)$ could just "touch" the $E=0$ value instead of "crossing" it. Therefore, it is necessary to check that level crossing really occurs. This can be done by calculating the time-gradient of the energy eigenvalue $E(t)$ at $t=t^{*}$. If $\mathrm{d} E /\left.\mathrm{d} t\right|_{t=t^{*}} \neq 0$, then there is level crossing at $t=t^{*}$.

The overall effect of level crossings can be characterized by the "spectral flow" $\mathcal{F}\left[t_{f}, t_{i}\right]$, defined to be the number of eigenvalues of the Dirac Hamiltonian $H(t, r)$ that cross zero from below minus the number of eigenvalues that cross zero from above, for the time interval $\left[t_{i}, t_{f}\right]$ considered. The spectral flow will be discussed further in Sec. IV. Here, we continue the investigation of the zero-eigenvalue equation per se.

\section{B. Gauge-invariant zero-eigenvalue equation}

We first express the zero-eigenvalue equation (3.2a) at a fixed time $t$ in terms of a set of bosonic background fields that are invariant under the $U(1)$ gauge transformations (2.11). As can be seen from Eq. (3.2), a nonvanishing gauge field $a_{0}$ contributes only a complex phase factor to the solution $\Psi$. Up to an overall phase factor, the solution is then

$$
\Psi(t, r)=\exp \left[-i \int_{r_{0}}^{r} \mathrm{~d} r^{\prime} a_{0}\left(t, r^{\prime}\right) / 2\right] \Psi_{\mathrm{M}}(t, r)
$$

provided $\Psi_{\mathrm{M}}(t, r)$ satisfies the nontrivial matrix equation

$$
\partial_{r} \Psi_{\mathrm{M}}=\left[-i \gamma_{5} a_{1} / 2-\left(\gamma^{1} \operatorname{Re} \chi+i \gamma^{0} \operatorname{Im} \chi\right) / r\right] \Psi_{\mathrm{M}}
$$

with boundary condition

$$
\Psi_{\mathrm{M}}(t, 0)=0
$$


The existence of a fermion zero mode for the linear differential equation (3.2) is thus equivalent to having a normalizable solution of Eq. (3.5) with boundary condition (3.6).

Next, we apply a unitary transformation to Eq. (3.5),

$$
\Psi_{\mathrm{M}} \rightarrow \Psi_{\Lambda} \equiv \Lambda^{\dagger} \Psi_{\mathrm{M}}
$$

with the transformation matrix

$$
\Lambda=-i \gamma^{0} \exp \left[i \varphi \gamma_{5} / 2\right]
$$

that diagonalizes the Hermitian matrix $\mathcal{A}_{H}$ via $\mathcal{A}_{H} \rightarrow \Lambda^{\dagger} \mathcal{A}_{H} \Lambda$. We obtain the following zero-eigenvalue equation for $\Psi_{\Lambda}$ :

$$
\begin{aligned}
\partial_{r} \Psi_{\Lambda} & =\left(\mathcal{A}_{0}+\mathcal{A}_{1}\right) \Psi_{\Lambda}, \\
\mathcal{A}_{0} & \equiv \Lambda^{\dagger} \mathcal{A}_{H} \Lambda=\lambda \gamma^{1}=-\lambda \sigma^{3} \\
\mathcal{A}_{1} & \equiv \Lambda^{\dagger}\left(-\partial_{r}-i \gamma_{5} a_{1} / 2\right) \Lambda=\mathcal{R} i \gamma_{5}=\mathcal{R} i \sigma^{2}
\end{aligned}
$$

with the further definitions

$$
\lambda \equiv \rho / r \geq 0, \quad \mathcal{R} \equiv\left(a_{1}-\partial_{r} \varphi\right) / 2
$$

It follows immediately from the definition (2.15) that the matrices $\mathcal{A}_{0}$ and $\mathcal{A}_{1}$ are invariant under the $U(1)$ gauge transformations (2.11). Moreover, $\mathcal{A}_{0}$ and $\mathcal{A}_{1}$ are real matrices and the solution $\Psi_{\Lambda}$ can be taken real, up to an overall complex phase factor. In the following, we take $\Psi_{\Lambda}$ to be strictly real and drop the subscript $\Lambda$.

For finite-energy background gauge fields with $r$-parity expansions as given by Eq. (2.7), one can show that the following limits hold:

$$
\lim _{r \rightarrow 0} \mathcal{R} / \lambda=\lim _{r \rightarrow \infty} \mathcal{R} / \lambda=0 .
$$

This demonstrates that the diagonal matrix $\mathcal{A}_{0}$ determines the local structure of the solution of the differential equation (3.9) in the regions of small and large $r$ (see also Ref. [19]).

\section{Spinor twist number and twist factor}

Since the solution $\Psi$ of the transformed zero-eigenvalue equation (3.9) is taken to be real, one can write $\Psi$ in polar notation,

$$
\Psi(t, r) \equiv|\Psi(t, r)| \exp \left[i \gamma_{5} \Theta(t, r)\right]\left(\begin{array}{l}
0 \\
1
\end{array}\right)
$$

where $\Theta \in \mathbb{R}$ measures the relative rotation of the spinor away from the $\Psi_{2}$-axis in the configuration space of $\Psi$. Recall that $\gamma_{5}=\sigma^{2}$, so that the exponential factor in Eq. (3.12) reads $11 \cos \Theta+i \sigma^{2} \sin \Theta$.

From Eqs. (3.9) and (3.12), one finds that $\Theta$ and $|\Psi|$ at fixed time $t$ satisfy the following coupled differential equations: 


$$
\begin{aligned}
\partial_{r} \Theta & =\mathcal{D}[\Theta]+\mathcal{R}, \\
\partial_{r}|\Psi| & =\lambda|\Psi| \cos 2 \Theta
\end{aligned}
$$

with the definitions

$$
\begin{aligned}
\mathcal{D}[\Theta] & \equiv-\lambda \sin 2 \Theta, \quad \mathcal{R} \equiv\left(a_{1}-\partial_{r} \varphi\right) / 2, \\
\lambda & \equiv \rho / r \geq 0
\end{aligned}
$$

In order to obtain regular behavior at $r=0$, the solutions of the differential equations (3.13) must satisfy the following boundary conditions:

$$
\begin{aligned}
\Theta(t, 0) & =0 \bmod \pi \\
|\Psi(t, 0)| & =0
\end{aligned}
$$

More specifically, these boundary conditions are needed because $\lambda$ is singular at the spatial origin $r=0$; see Eq. (2.14).

The nonlinearity of the differential equation (3.13a) originates from the fact that the linear differential equation (3.9) mixes the components of the spinor $\Psi$. Furthermore, the differential equation (3.13a) involves only $\Theta$, whereas Eq. (3.13b) contains both $\Theta$ and $|\Psi|$. These two properties of Eq. (3.13a) will turn out to be crucial for the results of the present paper.

Remarkably, the nonlinear differential equation (3.13a) for a given time slice $t$ can be transformed into a generalized Riccati equation 14,15 by setting $Y(t, r)=\tan \Theta(t, r)$,

$$
\partial_{r} Y-\mathcal{R}\left(1+Y^{2}\right)+2 \lambda Y=0 \text {. }
$$

The analysis is, however, best carried out with the nonlinear differential equation in the form as given by Eq. (3.13a), where the term $\mathcal{D}$ is called the "deviator" and the term $\mathcal{R}$ the "rotator," for reasons that will become clear shortly. Henceforth, we refer to the single differential equation (3.13a), with the implicit boundary condition (3.15a), as the "transformed Riccati equation."

Let us consider the asymptotic behavior of the solution $\Theta(t, r)$ of the transformed Riccati equation (3.13a) at a fixed time $t$. The deviator $\mathcal{D}$ dominates, in general, the right-hand side of Eq. (3.13a) for large $r$, according to Eq. (3.11). For large $r$, Eq. (3.13a) can therefore be approximated by

$$
\partial_{r} \Theta=-\lambda \sin 2 \Theta
$$

The differential equation (3.17) has three types of solutions at a fixed time slice $t$,

$$
\begin{aligned}
\Theta(t, r)= & N \pi, \\
\Theta(t, r)= & \left(N^{\prime}+1 / 2\right) \pi, \\
\tan [\Theta(t, r)]= & \tan \left[\Theta\left(t, r_{0}\right)\right] \\
& \times \exp \left[-2 \int_{r_{0}}^{r} \mathrm{~d} r^{\prime} \lambda\left(t, r^{\prime}\right)\right],
\end{aligned}
$$


for arbitrary integers $N$ and $N^{\prime}$.

The nontrivial solution $\Theta(t, r)$ given by Eq. (3.18d) is attracted toward the value $N \pi$ as $r \rightarrow \infty$, since $\lambda(t, r)$ is non-negative and has a divergent integral toward infinity. This shows that the "point" $\Theta(t, r)=N \pi$, with $N \in \mathbb{Z}$, is asymptotically stable in the solution space of the differential equation (3.17); cf. Ref. [19]. For the trivial solution $\Theta(t, r)=\left(N^{\prime}+1 / 2\right) \pi$, an arbitrarily small deviation will lead to a nontrivial solution given by Eq. (3.180), which asymptotically approaches the value $N^{\prime \prime} \pi$, with $N^{\prime \prime} \in \mathbb{Z}$. The "point" $\Theta(t, r)=\left(N^{\prime}+\right.$ $1 / 2) \pi$, with $N^{\prime} \in \mathbb{Z}$, is thus asymptotically unstable in the solution space of the differential equation (3.17).

The solutions of the complete differential equation 3.13a with boundary condition (3.15a) can thus be classified according to their asymptotic behavior. At a fixed time slice $t$, there are two classes:

$$
\begin{aligned}
S_{N}(t) & \equiv\left\{\Theta(t, r) \mid \lim _{r \rightarrow \infty} \Theta(t, r)=N \pi\right\}, \\
U_{N^{\prime}}(t) & \equiv\left\{\Theta(t, r) \mid \lim _{r \rightarrow \infty} \Theta(t, r)=\left(N^{\prime}+1 / 2\right) \pi\right\},
\end{aligned}
$$

with $N, N^{\prime} \in \mathbb{Z}$. If $\Theta \in S_{N}\left(\Theta \in U_{N}\right)$, then $\Theta$ is asymptotically stable (unstable) in the solution space of the differential equation (3.13a).

For any solution $\Theta(t, r) \in U_{N}(t)$ with arbitrary integer $N$, there necessarily exists a fermion zero mode, as follows from Eqs. (3.13b) and (3.15b). [It is clear that the normalizability condition (3.3) of the fermion zero mode requires the asymptotics of Eq. (3.19b).] Therefore, it suffices to study the transformed Riccati equation (3.13a) in order to determine the existence of a fermion zero mode at a particular time $t$.

At this moment, we can explain the use of the terms "deviator" and "rotator" in the transformed Riccati equation (3.13a). The observation from Eqs. (3.17) and (3.180) is that $\mathcal{D}$ pulls $\Theta(t, r)$ toward the value $N \pi$ as $r \rightarrow \infty$. In other words, it leads to a deviation of $\Theta(t, r)$ from the special path approaching the value $\left(N^{\prime}+1 / 2\right) \pi$ as $r \rightarrow \infty$, for which a fermion zero mode exists. This is the reason for calling the term $\mathcal{D}$ in Eq. (3.13a the "deviator." In the absence of the deviator $\mathcal{D}$ over the interval $\left[r_{0}, r_{1}\right]$, say, one observes from Eq. (3.13a) that $\mathcal{R}$ generates a simple rotation of the spinor by the angle $\Delta \Theta=\int_{r_{0}}^{r_{1}} \mathrm{~d} r^{\prime} \mathcal{R}\left(r^{\prime}\right)$. This is then the reason for calling the term $\mathcal{R}$ in Eq. (3.13a) the "rotator." The fermion zero mode solutions will be discussed further in the next subsection. Here, we continue the discussion of the transformed Riccati equation from a more general viewpoint.

Using analyticity and the Cauchy-Lipschitz existence and uniqueness theorem for ordinary differential equations [14,15,19, it can be shown that the solution $\Theta(t, r)$ of the transformed Riccati equation (3.13a) with boundary condition $\Theta(t, 0)=0$ is unique. For the regular finite-energy gauge fields considered, it can also be shown that the solution $\Theta(t, r)$ is bounded.

The uniqueness of the solution $\Theta(t, r)$ and its asymptotic behavior allow us to classify the gauge field background at one particular time $t$ by the quantity

$$
N_{\Theta}(t) \equiv[\Theta(t, \infty)-\Theta(t, 0)] / \pi,
$$

which can take integer or half-odd-integer values. According to the definition (3.12), the mapping $\mathcal{G}(t, r) \equiv \exp \left[i \gamma_{5} \Theta(t, r)\right] \in S O(2)$ gives the twisting of the spinor in the configuration space of $\Psi$ for fixed time $t$. We therefore call $N_{\Theta}(t)$ the "spinor twist number." 
It is now convenient to characterize a time-dependent spherically symmetric $S U(2)$ gauge field background by the change of spinor twist number between initial and final configurations. Henceforth, we call

$$
\Delta N_{\Theta}\left[t_{f}, t_{i}\right] \equiv N_{\Theta}\left(t_{f}\right)-N_{\Theta}\left(t_{i}\right)
$$

the "twist factor" of the spherically symmetric $S U(2)$ gauge field over the time interval $\left[t_{i}, t_{f}\right]$, with $t_{i}<t_{f}$.

It is important to realize that the twist factor $\Delta N_{\Theta}$ measures an intrinsic property of the $S U(2)$ gauge field configuration. Formally, Eqs. (3.9a), (3.12), (3.20), and (3.21) give

$$
\begin{aligned}
\Delta N_{\Theta}\left[t_{f}, t_{i}\right] & =\frac{1}{\pi} \int_{0}^{\infty} \mathrm{d} r \int_{t_{i}}^{t_{f}} \mathrm{~d} t \frac{\partial}{\partial t}\left(\frac{\partial}{\partial r} \Theta(t, r)\right), \\
\Theta(t, r) & \equiv-\frac{1}{2} \operatorname{Tr}\left(i \sigma^{2} \ln \left\{\lim _{\epsilon \rightarrow 0} \epsilon \mathcal{P} \exp \left[\int_{\epsilon}^{r} \mathrm{~d} r^{\prime}\left\{\mathcal{A}_{0}\left(t, r^{\prime}\right)+\mathcal{A}_{1}\left(t, r^{\prime}\right)\right\}\right]\right\}\right),
\end{aligned}
$$

where $\mathcal{P}$ represents path ordering. Here, $\mathcal{A}_{0}(t, r)$ and $\mathcal{A}_{1}(t, r)$ are defined by Eqs. (3.9b,c), in terms of the (1+1)-dimensional gauge field functions $\rho(t, r), \varphi(t, r)$, and $a_{1}(t, r)$. Whether or not there exists a more direct way to obtain $\Delta N_{\Theta}$ remains an open question.

\section{Necessary condition for fermion zero modes}

With the results of the previous subsection, it is possible to find a necessary condition for the existence of fermion zero modes at a particular time $t$. We first introduce the following diagnostic:

$$
\begin{aligned}
\mathcal{K}_{ \pm}(t) & \equiv \int_{D_{ \pm}(t)} \mathrm{d} r \mathcal{R}(t, r) \\
& \equiv \int_{0}^{\infty} \mathrm{d} r \theta[ \pm \mathcal{R}(t, r)] \mathcal{R}(t, r),
\end{aligned}
$$

with the domains of positive or negative values of $\mathcal{R}(t, r)$ defined by

$$
D_{ \pm}(t) \equiv\{r \mid \operatorname{sgn}[\mathcal{R}(t, r)]= \pm 1\} \subseteq[0, \infty)
$$

and $\theta$ the usual step function, $\theta[x]=0$ for $x<0$ and $\theta[x]=1$ for $x>0$. Note that, by definition, $\mathcal{K}_{+} \geq 0$ and $\mathcal{K}_{-} \leq 0$. Note also that the rotator $\mathcal{R}(t, r)$ from Eq. (3.14) is entirely defined in terms of the background fields $a_{1}(t, r)$ and $\varphi(t, r) \equiv \arg \chi(t, r)$.

Consider the transformed Riccati equation (3.13a) with boundary condition $\Theta(t, 0)=0$. The integration of $\mathcal{R}(t, r)$ over the domain $D_{+}\left(D_{-}\right)$then accounts for the rotation of the spinor in the "+" " -") direction. But the deviator $\mathcal{D}$, for values $\Theta \in(-\pi / 2, \pi / 2)$, brakes the rotation forced by the rotator $\mathcal{R}$. The crucial point, now, is that in order to have a fermion zero mode at time $t$ the total action of the rotator should overcome the resistance from the deviator in the region $-\pi / 2<\Theta<\pi / 2$, so that the solution $\Theta(t, r)$ ends up with $|\Theta| \geq \pi / 2$ at $r=\infty$. A necessary condition for the existence of a fermion zero mode at one particular time $t$ is, therefore, 


$$
\mathcal{K}_{\max }(t) \equiv \max \left[\mathcal{K}_{+}(t),\left|\mathcal{K}_{-}(t)\right|\right] \geq \pi / 2
$$

Having established the necessary condition (3.25), it would certainly be interesting to obtain also a necessary and sufficient condition for the existence of a fermion zero mode in a given static gauge field background. But, without further input, it appears difficult to find such a condition. For this reason, we turn in the next section to the role of time-dependent, continuous gauge field backgrounds.

\section{SPECTRAL FLOW}

In this section, we consider the spectrum of the effective Dirac Hamiltonian (3.1b) for time-dependent spherically symmetric $S U(2)$ gauge fields. In Sec. IV A, we derive a relation, Eq. (4.32), between level crossing and the change of winding number or spinor twist number over an infinitesimal time interval. From this result, we obtain in Sec. IV B the appropriate relation, Eq. (4.39), for the spectral flow over a finite time interval. Section IV A is rather technical and may be skipped on a first reading.

\section{A. Level crossing from changes in winding and twist numbers}

\section{Perturbative expansion}

We start from the transformed Riccati equation (3.13a), with boundary condition (3.15a), at a particular time $t=t^{*}$ and study the change of the solution $\Theta(t, r)$ in the neighborhood of $t=t^{*}$. For finite $r$ and $t=t^{*} \pm \epsilon$ with $\epsilon$ an arbitrarily small positive constant, one can expand the background fields as follows

$$
\begin{gathered}
\lambda\left(t^{*} \pm \epsilon, r\right)=\lambda\left(t^{*}, r\right) \pm\left.\epsilon \partial_{t} \lambda\right|_{t=t_{ \pm}^{*}}+\mathrm{O}\left(\epsilon^{2}\right), \\
\mathcal{R}\left(t^{*} \pm \epsilon, r\right)=\mathcal{R}\left(t^{*}, r\right) \pm\left.\epsilon \partial_{t} \mathcal{R}\right|_{t=t_{ \pm}^{*}}+\mathrm{O}\left(\epsilon^{2}\right),
\end{gathered}
$$

where the upper and lower time derivatives of the background fields are defined by

$$
\begin{aligned}
& \left.\partial_{t} \lambda(t, r)\right|_{t=t_{+}^{*}} \equiv \lim _{t \downarrow t^{*}} \partial_{t} \lambda(t, r), \\
& \left.\partial_{t} \lambda(t, r)\right|_{t=t_{-}^{*}} \equiv \lim _{t \uparrow t^{*}} \partial_{t} \lambda(t, r),
\end{aligned}
$$

and similarly for $\partial_{t} \mathcal{R}$. The solution $\Theta(t, r)$ for $t=t^{*} \pm \epsilon$ can be written as

$$
\Theta\left(t^{*} \pm \epsilon, r\right)=\Theta_{ \pm}\left(t^{*}, r\right) \pm \epsilon f_{1}\left(t^{*}, r\right)+\mathrm{O}\left(\epsilon^{2}\right)
$$

The function $f_{1}(t, r)$ is continuous at $t=t^{*}$, but the first term on the right-hand side of Eq. (4.3) allows for a discontinuity. For the moment, we consider the functions $\Theta_{ \pm}\left(t^{*}, r\right)$ in Eq. (4.3) to be equal.

By substituting Eqs. (4.1) and (4.3) into the transformed Riccati equation (3.13a), one obtains to first order in $\epsilon$ the following linear differential equation for $f_{1}$ : 


$$
\left[\partial_{r}+2 \lambda\left(t^{*}, r\right) \cos 2 \Theta\left(t^{*}, r\right)\right] f_{1}\left(t^{*}, r\right)=j_{1}\left(t^{*}, r\right)
$$

with the definition

$$
\left.j_{1}\left(t^{*}, r\right) \equiv \partial_{t} \mathcal{R}\right|_{t=t^{*}}-\left.\partial_{t} \lambda\right|_{t=t^{*}} \sin 2 \Theta
$$

and boundary condition

$$
f_{1}\left(t^{*}, 0\right)=0
$$

The solution of the differential equation (4.4) is found to be

$$
f_{1}\left(t^{*}, r\right)=\mathcal{J}_{1}\left(t^{*}, r\right) /\left|\Psi\left(t^{*}, r\right)\right|^{2}
$$

with the definition

$$
\mathcal{J}_{1}\left(t^{*}, r\right) \equiv \int_{0}^{r} \mathrm{~d} r^{\prime}\left|\Psi\left(t^{*}, r^{\prime}\right)\right|^{2} j_{1}\left(t^{*}, r^{\prime}\right) .
$$

Here, we have used the solution of Eq. (3.13b),

$$
|\Psi(t, r)| \propto \exp \left[\int_{r_{0}}^{r} \mathrm{~d} r^{\prime} \lambda\left(t, r^{\prime}\right) \cos 2 \Theta\left(t, r^{\prime}\right)\right],
$$

to obtain Eq. (4.7) in the form shown.

The function $j_{1}(t, r)$ is continuous at $t=t^{*}$ for smooth background fields $\lambda(t, r)$ and $\mathcal{R}(t, r)$, which implies that the solution $f_{1}(t, r)$ of Eq. (4.4) is also continuous at $t=t^{*}$. If, on the other hand, the time slice $t=t^{*}$ corresponds to a local change of the gauge field winding number $N_{\chi}$, the partial derivatives of $\lambda(t, r)$ and $\mathcal{R}(t, r)$ are not well-defined at $t=t^{*}$, as will be shown later. In this case, the function $j_{1}(t, r)$ is not well-defined either, which affects the continuity of the solution $f_{1}(t, r)$ of Eq. (4.4). In Sec. IV A 3, we will show that the function $f_{1}(t, r)$ can be taken to be continuous at $t=t^{*}$, provided possible discontinuities are accounted for by the leading terms $\Theta_{-}\left(t^{*}, r\right)$ and $\Theta_{+}\left(t^{*}, r\right)$ in Eq. (4.3).

\section{Time-differentiable $\lambda$ and $\mathcal{R}$}

Consider a particular time slice $t=t^{*}$, for which the $\lambda(t, r)$ and $\mathcal{R}(t, r)$ fields are differentiable with respect to time,

$$
\begin{aligned}
\left.\partial_{t} \lambda(t, r)\right|_{t=t_{+}^{*}} & =\left.\partial_{t} \lambda(t, r)\right|_{t=t_{-}^{*}}, \\
\left.\partial_{t} \mathcal{R}(t, r)\right|_{t=t_{+}^{*}} & =\left.\partial_{t} \mathcal{R}(t, r)\right|_{t=t_{-}^{*}}
\end{aligned}
$$

with upper and lower time derivatives as defined in Eq. (4.2).

First, suppose that there is no fermion zero mode at $t=t^{*}$, so that $\Theta\left(t^{*}, r\right) \rightarrow N \pi$ as $r \rightarrow \infty$. For large $r$ and using Eq. (3.11), one then obtains from Eq. (4.4) the results $\lim _{r \rightarrow \infty} j_{1} / \lambda=0$ and $f_{1} \propto r^{-2}$, which imply

$$
\lim _{r \rightarrow \infty} f_{1}\left(t^{*}, r\right)=0
$$


This indicates that there is no change of the asymptotic behavior of $\Theta(t, r)$ in the neighborhood of $t=t^{*}$, which corresponds to having a constant spinor twist number (3.20) at $t=t^{*}$

$$
\left.\delta N_{\Theta}\right|_{t=t^{*}} \equiv N_{\Theta}\left(t^{*}+\epsilon\right)-N_{\Theta}\left(t^{*}-\epsilon\right)=0 .
$$

[We reserve the notation $\Delta N_{\Theta}$ for the global change of spinor twist number; see Eq. (3.21). Of course, $\delta N_{\Theta}$ is in no way "infinitesimal," see Eqs. (4.14) below.]

Next, consider the case of having a normalized fermion zero mode at $t=t^{*}$ with $\Theta\left(t^{*}, r\right) \in U_{N}$, that is, belonging to the "unstable" class of solutions (3.19b). Since the solution $\Theta\left(t^{*}, r\right) \in U_{N}$ is asymptotically unstable, one observes from Eq. (3.18d) that a small positive [negative] perturbation of $\Theta$ at large $r$ leads to $\Theta\left(t^{*}, r\right) \in S_{N+1}\left[\Theta\left(t^{*}, r\right) \in S_{N}\right]$. From Eqs. (4.3), (4.7), and (4.8) one deduces that the fermion zero mode at $t=t^{*}$ sits at a bifurcation point for different $N_{\Theta}$ 's. In fact, the local change of spinor twist number

$$
\left.\delta N_{\Theta}\right|_{t=t^{*}} \equiv N_{\Theta}\left(t^{*}+\epsilon\right)-N_{\Theta}\left(t^{*}-\epsilon\right)
$$

is given by

$$
\left.\delta N_{\Theta}\right|_{t^{*}}=\left\{\begin{array}{lll}
+1 & \text { for } & \mathcal{J}_{1}\left(t^{*}, \infty\right)>0, \\
-1 & \text { for } & \mathcal{J}_{1}\left(t^{*}, \infty\right)<0,
\end{array}\right.
$$

as long as $\mathcal{J}_{1}\left(t^{*}, \infty\right) \neq 0$. [The special case of $\mathcal{J}_{1}\left(t^{*}, \infty\right)=0$ will be discussed in Sec. IV A 4.] For an elementary discussion of bifurcation theory; see Ref. [19].

Having a fermion zero mode at $t=t^{*}$, we are especially interested in the time-gradient of the fermion energy eigenvalue at $t=t^{*}$, in order to check for level crossing. The timegradient of the energy eigenvalue of the Dirac Hamiltonian at $t=t^{*}$ is calculated up to the first order in $\epsilon$ :

$$
\begin{aligned}
\left.\frac{\mathrm{d} E}{\mathrm{~d} t}\right|_{t=t^{*}} & =\left\langle\Psi_{l}\left(t^{*}, r\right)\left|\frac{\partial H}{\partial t}\left(t^{*}\right)\right| \Psi_{l}\left(t^{*}, r\right)\right\rangle \\
& =\mathcal{J}_{1}\left(t^{*}, \infty\right),
\end{aligned}
$$

where $\mathcal{J}_{1}$ is defined by Eq. (4.8) and $\Psi_{l}\left(t^{*}, r\right)$ represents the (nondegenerate) normalized fermion zero mode at $t=t^{*}$ in the two-component spinor notation of Eq. (2.9). The expectation value used in Eq. (4.15) is defined by

$$
\begin{aligned}
& \left\langle\Psi_{l}\left(t^{*}, r\right)\left|O\left(t^{*}\right)\right| \Psi_{l}\left(t^{*}, r\right)\right\rangle \\
& \equiv \int_{0}^{\infty} \mathrm{d} r \Psi_{l}\left(t^{*}, r\right)^{\dagger} O\left(t^{*}\right) \Psi_{l}\left(t^{*}, r\right),
\end{aligned}
$$

for an arbitrary time-dependent Hermitian operator $O(t)$.

From Eqs. (4.14) and (4.15), we obtain

$$
\operatorname{sgn}\left[\left.\frac{\mathrm{d} E}{\mathrm{~d} t}\right|_{t=t^{*}}\right]=\left.\delta N_{\Theta}\right|_{t=t^{*}} \in\{-1,+1\},
$$

with the implicit limit $\epsilon \rightarrow 0$ on the right-hand side. This establishes the relation between level crossing and the change of spinor twist number, for the case that $\lambda(t, r)$ and $\mathcal{R}(t, r)$ are time-differentiable at $t=t^{*}$ and generic for times close to it [so that $\mathcal{J}_{1}\left(t^{*}, \infty\right) \neq 0$ ]. 


\section{Time-nondifferentiable $\lambda$ and $\mathcal{R}$}

Now, consider a gauge field background for which $\chi(t, r)$ vanishes at the spacetime point $\left(t^{*}, r^{*}\right)$ and the winding number $N_{\chi}$ as defined in Eq. (2.16) changes from a value $N$ to $N+\left.\delta N_{\chi}\right|_{t=t^{*}}$. Generally, the fields $\lambda(t, r)$ and $\mathcal{R}(t, r)$ are not differentiable with respect to time:

$$
\begin{gathered}
\left.\partial_{t} \lambda(t, r)\right|_{t=t_{+}^{*}} \neq\left.\partial_{t} \lambda(t, r)\right|_{t=t_{-}^{*}}, \\
\left.\partial_{t} \mathcal{R}(t, r)\right|_{t=t_{+}^{*}} \neq\left.\partial_{t} \mathcal{R}(t, r)\right|_{t=t_{-}^{*}},
\end{gathered}
$$

with upper and lower time derivatives as defined in Eq. (4.2).

Let us have a closer look at the discontinuities of the time derivatives of $\lambda(t, r)$ and $\mathcal{R}(t, r)$ at $t=t^{*}$. First, one observes from the definition (3.14) of the $\lambda(t, r)$ field that the time derivative of $\lambda(t, r)$ is not well-defined at the spacetime point $\left(t^{*}, r^{*}\right)$ :

$$
\begin{aligned}
\left.\partial_{t} \lambda\left(t, r^{*}\right)\right|_{t=t_{+}^{*}} & =-\left.\partial_{t} \lambda\left(t, r^{*}\right)\right|_{t=t_{-}^{*}} \\
& =\left|\partial_{t} \chi\left(t^{*}, r^{*}\right)\right| / r^{*} .
\end{aligned}
$$

Second, introduce the gauge-invariant function $\mathrm{R}\left(t^{*}, r\right)$ defined by

$$
\begin{aligned}
\mathrm{R}\left(t^{*}, r\right) & \equiv \lim _{\epsilon \rightarrow 0}\left[\mathcal{R}\left(t^{*}+\epsilon, r\right)-\mathcal{R}\left(t^{*}-\epsilon, r\right)\right] \\
& =\lim _{\epsilon \rightarrow 0}\left[\left.\partial_{r} \varphi\right|_{t=t^{*}-\epsilon}-\left.\partial_{r} \varphi\right|_{t=t^{*}+\epsilon}\right] / 2 .
\end{aligned}
$$

Taylor expanding $\partial_{r} \varphi(t, r)$ with respect to both $t$ and $r$ in the vicinity of the spacetime point $\left(t^{*}, r^{*}\right)$ and using the fact that $\alpha(t, r) \rightarrow 0$ and $\beta(t, r) \rightarrow 0$ as $(t, r) \rightarrow\left(t^{*}, r^{*}\right)$, one finds that $\mathrm{R}\left(t^{*}, r\right)$ shoots up to infinity at $r=r^{*}$, whereas it drops to zero for $r \neq r^{*}$. Taking the gauge condition $\chi(t, 0)=\chi(t, \infty)=1$, one readily proves that

$$
\int_{0}^{\infty} \mathrm{d} r \mathrm{R}\left(t^{*}, r\right)=-\left.\pi \lim _{\epsilon \rightarrow 0} \delta N_{\chi}\right|_{t=t^{*}},
$$

with the local change of winding number defined as

$$
\left.\delta N_{\chi}\right|_{t=t^{*}} \equiv N_{\chi}\left(t^{*}+\epsilon\right)-N_{\chi}\left(t^{*}-\epsilon\right) .
$$

This shows that the function $\mathrm{R}\left(t^{*}, r\right)$ is proportional to a Dirac delta-function centered at $r=r^{*}$,

$$
\mathrm{R}\left(t^{*}, r\right)=-\left.\pi \lim _{\epsilon \rightarrow 0} \delta N_{\chi}\right|_{t=t^{*}} \delta\left(r-r^{*}\right) .
$$

The nonvanishing right-hand side of Eq. (4.23) for $r=r^{*}$ implies that the time derivative of the rotator $\mathcal{R}(t, r)$ is not well-defined at the spacetime point $\left(t^{*}, r^{*}\right)$.

The delta-function-like behavior (4.23) of $\mathrm{R}$ can be used to derive the effect of the change of the gauge field winding number on the change of the spinor twist number at $t=t^{*}$. Start by defining

$$
\Theta_{-}\left(t^{*}, r\right) \equiv \lim _{t \uparrow t^{*}} \Theta(t, r)
$$


Then, if $\Theta_{-}\left(t^{*}, r\right)$ belongs to the class $S_{N}$, for some integer $N$, one deduces from Eqs. (3.13a), $(4.20)$, and 4.23$)$ that

$$
\begin{aligned}
\Theta_{+}\left(t^{*}, r\right) & \equiv \lim _{t \downarrow t^{*}} \Theta(t, r) \\
& =\Theta_{-}\left(t^{*}, r\right)-\left.\pi \lim _{\epsilon \rightarrow 0} \delta N_{\chi}\right|_{t=t^{*}} \theta\left(r-r^{*}\right),
\end{aligned}
$$

where $\theta$ is the usual step function. This implies that the solution changes from one class to another, as $t$ crosses the value $t^{*}$. Specifically, if the earlier solution $\Theta_{-}\left(t^{*}, r\right)$ belongs to $S_{N}$, then the later solution $\Theta_{+}\left(t^{*}, r\right)$ belongs to $S_{N-\delta N_{\chi}}$.

Equation (4.25) shows that the change of the gauge field winding number $\left.\delta N_{\chi}\right|_{t=t^{*}}$ causes the change of the spinor twist number at $t=t^{*}$ to be given by

$$
\left.\delta N_{\Theta ; \mathrm{A}}\right|_{t=t^{*}}=-\left.\delta N_{\chi}\right|_{t=t^{*}}
$$

regardless of the existence of a fermion zero mode at $t=t^{*}$ (this contribution is labeled A).

Before we continue with the evaluation of the change of spinor twist number, we need to address the continuity issue for the solution $f_{1}$ of Eq. (4.4). According to Eqs. (4.19) and (4.23), the time derivatives of the gauge-invariant $\lambda(t, r)$ and $\mathcal{R}(t, r)$ fields are not welldefined at the spacetime point $\left(t^{*}, r^{*}\right)$. This implies that the function $j_{1}(t, r)$, as defined in Eq. (4.5), does not have a well-defined value either. Note that all these problems can be traced to the discontinuity of the unitary matrix $\Lambda$ in Eq. (3.8), which, in turn, is caused by the ill-defined argument $\varphi(t, r)$ of the field $\chi(t, r)$ at the spacetime point $\left(t^{*}, r^{*}\right)$ where $\chi(t, r)$ vanishes. By performing the inverse unitary transformation of Eq. (3.7), $\Psi \rightarrow \Psi_{l} \equiv \Lambda \Psi$, one finds that the $j_{1}$ "expectation value" (4.8) is again given by

$$
\mathcal{J}_{1}\left(t^{*}, R\right)=\left\langle\Psi_{l}\left(t^{*}, r\right)\left|\frac{\partial H}{\partial t}\left(t^{*}\right)\right| \Psi_{l}\left(t^{*}, r\right)\right\rangle_{R},
$$

with the implicit integral over $r$ on the right-hand side running over $[0, R]$. The right-hand side of Eq. (4.27) has a well-defined value at $t=t^{*}$ for smooth background fields $a_{0}(t, r)$, $a_{1}(t, r)$, and $\chi(t, r)$. This shows that the discontinuity of the function $j_{1}(t, r)$ at $\left(t^{*}, r^{*}\right)$, caused by an ill-defined function $\varphi(t, r)$, can be absorbed into the local change of the spinor twist number $\left.\delta N_{\Theta ; \mathrm{A}}\right|_{t=t^{*}}$ via the relation (4.26).

With the discontinuity of $j_{1}(t, r)$ at $\left(t^{*}, r^{*}\right)$ absorbed into the local change of the spinor twist number $\left.\delta N_{\Theta ; \mathrm{A}}\right|_{t=t^{*}}$, the solution $f_{1}(t, r)$ of Eq. (4.4), explicitly given by Eq. (4.7), takes a well-defined value at $t=t^{*}$ and may produce a local change of spinor twist number that is not associated with the local change of gauge field winding number.

Finally, we are ready to consider the additional effect on the local change of the spinor twist number due to the presence of a fermion zero mode at $t=t^{*}$ (this contribution will be labeled B). Since $\mathcal{J}_{1}\left(t^{*}, \infty\right)$ as given by Eq. (4.27) has a well-defined value at $t=t^{*}$, we have a unique time gradient for the level crossing,

$$
\left.\frac{\mathrm{d} E}{\mathrm{~d} t}\right|_{t=t^{*}+\epsilon}=\left.\frac{\mathrm{d} E}{\mathrm{~d} t}\right|_{t=t^{*}-\epsilon}=\mathcal{J}_{1}\left(t^{*}, \infty\right) .
$$

Now, generic background gauge fields with $\mathcal{J}_{1}\left(t^{*}, \infty\right) \neq 0$ produce the following change of spinor twist number at $t=t^{*}$ [see Eqs. (4.14) above]: 


$$
\left.\delta N_{\Theta ; \mathrm{B}}\right|_{t=t^{*}}=\operatorname{sgn}\left[\mathcal{J}_{1}\left(t^{*}, \infty\right)\right],
$$

in addition to the contribution $\left.\delta N_{\Theta ; \mathrm{A}}\right|_{t=t^{*}}$ given by Eq. (4.26). [Remark that $\delta N_{\Theta ; \mathrm{B}}=$ \pm 1 for the generic case considered.] According to Eq. (4.28), the level crossing at $t=t^{*}$ is determined by $\operatorname{sgn}\left[\mathcal{J}_{1}\left(t^{*}, \infty\right)\right]$. We therefore deduce the following relation between level crossing and the change of the spinor twist number:

$$
\begin{aligned}
\operatorname{sgn}\left[\left.\frac{\mathrm{d} E}{\mathrm{~d} t}\right|_{t=t^{*}}\right] & =\left.\delta N_{\Theta ; \mathrm{B}}\right|_{t=t^{*}} \\
& =\left.\delta N_{\Theta}\right|_{t=t^{*}}-\left.\delta N_{\Theta ; \mathrm{A}}\right|_{t=t^{*}},
\end{aligned}
$$

where the total change of the spinor twist number at $t=t^{*}$ is given by the sum of both contributions

$$
\left.\delta N_{\Theta}\right|_{t=t^{*}}=\left.\delta N_{\Theta ; \mathrm{A}}\right|_{t=t^{*}}+\left.\delta N_{\Theta ; \mathrm{B}}\right|_{t=t^{*}} .
$$

Combining Eqs. (4.26) and (4.30), we find that the local spectral flow $\mathcal{F}\left[t^{*}+\epsilon, t^{*}-\epsilon\right]$ is given in terms of the local winding factor and twist factor,

$$
\mathcal{F}\left[t^{*}+\epsilon, t^{*}-\epsilon\right]=\left.\delta N_{\chi}\right|_{t=t^{*}}+\left.\delta N_{\Theta}\right|_{t=t^{*}},
$$

which is the main result of the present subsection. Here, $\delta N_{\chi}$ and $\delta N_{\Theta}$ are defined by Eqs. (4.22) and (4.13), respectively, and $\epsilon$ is a positive infinitesimal.

\section{Special and generic gauge field backgrounds}

Let us, finally, discuss the case of having a fermion zero mode at $t=t^{*}$, for which the "expectation value" $\mathcal{J}_{1}\left(t^{*}, \infty\right)$ vanishes,

$$
\begin{aligned}
\mathcal{J}_{1}\left(t^{*}, \infty\right) & \left.\equiv \int_{0}^{\infty} \mathrm{d} r\left|\Psi\left(t^{*}, r\right)\right|^{2}\left[\partial_{t} \mathcal{R}-\partial_{t} \lambda \sin 2 \Theta\right]\right|_{t=t^{*}} \\
& =0 .
\end{aligned}
$$

This implies $\mathrm{d} E /\left.\mathrm{d} t\right|_{t=t^{*}}=0$, according to Eq. (4.28). A nonvanishing $\mathrm{d}^{2} E /\left.\mathrm{d} t^{2}\right|_{t=t^{*}}$, with vanishing first-order derivative, now corresponds to the absence of level crossing (the fermion energy eigenvalue just touches $E=0$ at $t=t^{*}$ ). But a nonvanishing $\mathrm{d}^{3} E /\left.\mathrm{d} t^{3}\right|_{t=t^{*}}$, with vanishing first- and second-order derivatives, again has level crossing. One therefore needs to find the first nonvanishing derivative of the energy corresponding to the fermion zero mode, in order to determine whether or not level crossing occurs.

So far, we have considered only the solution $\Psi(t, r)$ of the zero-eigenvalue equation (3.2a), not the full spectrum (3.1.) of the time-dependent Dirac Hamiltonian. This makes it impossible to obtain the relation between the local change of the spinor twist number and the time derivatives of $E(t)$ at $t=t^{*}$ beyond the leading-order approximation. Still, the relation (4.32) can be shown to hold for generic $S U(2)$ gauge field backgrounds, since background fields with $\mathcal{J}_{1}\left(t^{*}, \infty\right)=0$ form a class of measure zero.

Start from the gauge-invariant background fields $\lambda(t, r)$ and $\mathcal{R}(t, r)$ at the time slice $t=t^{*}$ where the fermion zero mode resides, with profile functions $\Psi\left(t^{*}, r\right)$ and $\Theta\left(t^{*}, r\right)$. Under an 
infinitesimal time shift $t=t^{*} \rightarrow t^{*}+\epsilon$, generic $\lambda(t, r)$ and $\mathcal{R}(t, r)$ vary according to Eq. (4.1), with independent first-order coefficients (possible differences above and below $t=t^{*}$ are not important for the present argument). The change with time of these background fields is to first order in $\epsilon$

$$
\left.\delta \lambda \equiv \epsilon \partial_{t} \lambda\right|_{t=t^{*}},\left.\quad \delta \mathcal{R} \equiv \epsilon \partial_{t} \mathcal{R}\right|_{t=t^{*}}
$$

Next, define the following "expectation value" of $\delta \lambda$ :

$$
\begin{aligned}
<\delta \lambda>_{t=t^{*}} \equiv & \left.\int_{0}^{\infty} \mathrm{d} r\left|\Psi\left(t^{*}, r\right)\right|^{2} \delta \lambda(t, r)\right|_{t=t^{*}} \\
& \times \sin 2 \Theta\left(t^{*}, r\right),
\end{aligned}
$$

with an integration measure weighted by the known function $\sin 2 \Theta\left(t^{*}, r\right)$. The analogous "expectation value" of $\delta \mathcal{R}$ is

$$
<\delta \mathcal{R}>\left._{t=t^{*}} \equiv \int_{0}^{\infty} \mathrm{d} r\left|\Psi\left(t^{*}, r\right)\right|^{2} \delta \mathcal{R}(t, r)\right|_{t=t^{*}}
$$

but without extra weight function.

Now, recall that the functional $\mathcal{J}_{1}\left(t^{*}, \infty\right)$ as given by Eq. (4.33) is proportional to the difference of these "expectation values" in leading order,

$$
\begin{aligned}
\epsilon \mathcal{J}_{1}\left(t^{*}, \infty\right)= & <\delta \mathcal{R}>_{t=t^{*}}-<\delta \lambda>_{t=t^{*}} \\
& +\mathrm{O}\left(\epsilon^{2}\right) .
\end{aligned}
$$

Near the origin of the two-dimensional space spanned by $X \equiv<\delta \mathcal{R}>_{t=t^{*}}$ and $Y \equiv<$ $\delta \lambda>_{t=t^{*}}$, the class of background gauge fields with $\mathcal{J}_{1}\left(t^{*}, \infty\right)=0$ therefore coincides with the one-dimensional subspace

$$
\{(X, Y) \mid X=Y\}
$$

which is of measure zero. This shows that the quantity $\mathcal{J}_{1}\left(t^{*}, \infty\right)$ is nonzero for generic background gauge fields away from $t=t^{*}$ and that the relation between the local change of the spinor twist number and level crossing as given by Eqs. (4.17) and (4.30) holds in general.

\section{B. Relation between spectral flow and $S U(2)$ gauge field background}

The results of the previous subsection can be summarized as follows. For generic regular bosonic fields of the effective (1+1)-dimensional theory (2.8), the spectral flow $\mathcal{F}\left[t_{f}, t_{i}\right]$ for the time interval $\left[t_{i}, t_{f}\right]$ is given by the sum of the winding factor (2.17) and twist factor (3.21):

$$
\mathcal{F}\left[t_{f}, t_{i}\right]=\Delta N_{\chi}\left[t_{f}, t_{i}\right]+\Delta N_{\Theta}\left[t_{f}, t_{i}\right]
$$

This result is simply the grand total of all level crossings (4.32). 
As mentioned before, the spectral flow $\mathcal{F}$ in Eq. (4.39) is defined as the number of eigenvalues of the effective Dirac Hamiltonian (3.1b) that cross zero from below minus the number of eigenvalues that cross zero from above, for the time interval $\left[t_{i}, t_{f}\right]$ with $t_{i}<t_{f}$. The quantity $\mathcal{F}$ is an integer, by definition. But the winding factor $\Delta N_{\chi}$ and the twist factor $\Delta N_{\Theta}$ also take integer values in general.

Let us, nevertheless, discuss the special cases for which relation (4.39) is not applicable. The spectral flow $\mathcal{F}$ from Eq. (4.39) would not have a well-defined integer value if the gauge field winding number $N_{\chi}(t)$ or spinor twist number $N_{\Theta}(t)$ were ill-defined or noninteger at time slice $t \in \mathrm{T}_{i f} \equiv\left\{t_{i}, t_{f}\right\}$. In order to simplify the discussion, we exclude static field configurations from our considerations.

The gauge field winding number $N_{\chi}(t)$, in particular, is not well-defined for a time slice $t=t^{(1)} \in \mathrm{T}_{i f}$ if the function $\chi\left(t^{(1)}, r\right)$ has a zero, see Eqs. (2.15) and (2.16). Assume that the zero of $\chi$ occurs for $t^{(1)}=t_{i}$ and that this is the only problem. In this case, one can simply choose a real number $\delta t_{i}^{(1)}$, so that the field $\chi(t, r)$ has no zero at the time slice $t=t_{i}^{(1)} \equiv t_{i}+\delta t_{i}^{(1)}$. For the new time interval $\left[t_{i}^{(1)}, t_{f}\right]$, one then obtains an integer-valued winding factor $\Delta N_{\chi}\left[t_{f}, t_{i}^{(1)}\right]$. The other case of having the zero of $\chi$ at $t^{(1)}=t_{f}$ can be treated in the same way.

Alternatively, the spinor twist number $N_{\Theta}(t)$ can take a half-odd-integer value for time slice $t=t^{(2)} \in \mathrm{T}_{i f}$. Now recall that a half-odd-integer spinor twist number implies the existence of a fermion zero mode; see the paragraph below Eq. (3.19b). This makes it impossible to properly define the spectral flow for the exact time interval $\left[t_{i}, t_{f}\right]$. Assume that the zero mode occurs for $t^{(2)}=t_{i}$ and that this is the only problem. In this case, one can choose a real number $\delta t_{i}^{(2)}$, so that $N_{\Theta}(t)$ takes a well-defined integer value at the time slice $t=t_{i}^{(2)} \equiv t_{i}+\delta t_{i}^{(2)}$. This is always possible, because a fermion zero mode corresponds to an asymptotically unstable solution $\Theta$ of Eq. (3.13a). For the new time interval $\left[t_{i}^{(2)}, t_{f}\right]$, one then obtains an integer-valued twist factor $\Delta N_{\Theta}\left[t_{f}, t_{i}^{(2)}\right]$. The other case of having the zero mode at $t^{(2)}=t_{f}$ can be treated in the same way.

Henceforth, we assume generic spherically symmetric $S U(2)$ gauge field backgrounds, so that the right-hand side of Eq. (4.39) is well-defined and the sum of two integers. It is, furthermore, clear that the winding factor $\Delta N_{\chi}\left[t_{f}, t_{i}\right]$ is entirely determined by the background gauge fields [see Eqs. (2.15) and (2.16)]. But also the twist factor $\Delta N_{\Theta}\left[t_{f}, t_{i}\right]$ can be expressed solely in terms of background gauge fields [see Eq. (3.22)]. The relation (4.39) thus connects a property of the fermions, the spectral flow $\mathcal{F}$, to two characteristics of a generic spherically symmetric $S U(2)$ gauge field background, the winding and twist factors. This is the main result of the present paper.

\section{SPECTRAL FLOW FOR LÜSCHER-SCHECHTER $S U(2)$ GAUGE FIELDS}

In this section, we discuss the existence of fermion zero modes and the corresponding spectral flow for certain explicitly known time-dependent spherically symmetric solutions of the $S U(2)$ Yang-Mills equations. This allows for a nontrivial check of the relation (4.39) found in Sec. VD3. Throughout this section and in the figures, the same (arbitrary) mass scale is used to make the spacetime coordinates and energy dimensionless. 


\section{A. Brief review of the LS solutions}

The solutions considered in this section are spherically symmetric solutions of the $S U(2)$ gauge field equations, which describe collapsing and re-expanding shells of energy. The corresponding (1+1)-dimensional field equations from the reduced action (2.8a) read

$$
\begin{aligned}
& -\partial^{\mu}\left(r^{2} f_{\mu \nu}\right)=2 \operatorname{Im}\left(\chi^{*} D_{\nu} \chi\right), \\
& {\left[-D^{2}+\left(|\chi|^{2}-1\right) / r^{2}\right] \chi=0 .}
\end{aligned}
$$

Remarkably, Lüscher and Schechter were able to obtain analytic solutions of these coupled partial differential equations [12,13].

The Lüscher-Schechter (LS) solutions can be represented as follows (see Refs. [7],10] and references therein):

$$
\begin{aligned}
a_{\mu} & =-q(\tau) \partial_{\mu} w \\
\alpha & \equiv \operatorname{Re} \chi=1+q(\tau) \cos ^{2} w \\
\beta & \equiv \operatorname{Im} \chi=(1 / 2) q(\tau) \sin 2 w
\end{aligned}
$$

with the new coordinates

$$
\begin{aligned}
& \tau \equiv \operatorname{sgn}(t) \arccos \left(\frac{1+r^{2}-t^{2}}{\sqrt{\left(1+t^{2}-r^{2}\right)^{2}+4 r^{2}}}\right), \\
& w \equiv \arctan \left(\frac{1-r^{2}+t^{2}}{2 r}\right) .
\end{aligned}
$$

Using the Ansatz (5.2), the field equations (5.1) are reduced to a single nonlinear secondorder differential equation for $q(\tau)$,

$$
\frac{\mathrm{d}^{2} q}{\mathrm{~d} \tau^{2}}+2 q(q+1)(q+2)=0 .
$$

The ordinary differential equation (5.4) can be interpreted as belonging to a mechanical system consisting of a particle trapped in a double-well potential $V(q) \equiv \frac{1}{2} q^{2}(q+2)^{2}$. The conserved total energy $\epsilon$ of the particle trapped in the potential $V$ is then

$$
\epsilon=\frac{1}{2}\left(\frac{\mathrm{d} q}{\mathrm{~d} \tau}\right)^{2}+V(q), \quad V(q) \equiv q^{2}(q+2)^{2} / 2 .
$$

The general solution of Eq. (5.4) depends on the energy parameter $\epsilon$ and the $\tau$-translation parameter $\tau_{0}$, together with a further discrete parameter $\zeta= \pm 1$. The solutions of Eq. (5.4) can be divided into two classes, one with energy $\epsilon \leq 1 / 2$ and the other with energy $\epsilon>1 / 2$. Explicitly, the LS solutions are 12,13

$$
\begin{aligned}
& q(\tau)=-1+\zeta(1+\sqrt{2 \epsilon})^{1 / 2} \operatorname{dn}\left[(1+\sqrt{2 \epsilon})^{1 / 2}\left(\tau-\tau_{0}\right) \mid m^{-1}\right] \quad \text { for } \epsilon \leq 1 / 2, \\
& q(\tau)=-1+\zeta(1+\sqrt{2 \epsilon})^{1 / 2} \operatorname{cn}\left[(8 \epsilon)^{1 / 4}\left(\tau-\tau_{0}\right) \mid m\right] \quad \text { for } \epsilon>1 / 2,
\end{aligned}
$$


with the modulus defined by

$$
m \equiv \frac{1+\sqrt{2 \epsilon}}{2 \sqrt{2 \epsilon}} .
$$

Here, $\operatorname{dn}[u \mid m]$ and $\operatorname{cn}[u \mid m]$ are Jacobi elliptic functions [20,21.

For $\epsilon<1 / 2$, there exists no spacetime point where $\chi(t, r)$ vanishes. For $\epsilon \geq 1 / 2$, on the other hand, there are zeros of $\chi(t, r)$ at 10

$$
t_{n}=\tan \left(\tau_{0}+\frac{1+2 n}{(8 \epsilon)^{1 / 4}} K(m)\right), r_{n}=\sqrt{1+t_{n}^{2}},
$$

where $n$ is an integer that satisfies the condition

$$
-\frac{\pi}{2} \leq\left(\tau_{0}+\frac{1+2 n}{(8 \epsilon)^{1 / 4}} K(m)\right) \leq \frac{\pi}{2}
$$

Here, $K(m)$ is the complete elliptic integral of the first kind,

$$
K(m) \equiv \int_{0}^{1} \mathrm{~d} u\left[\left(1-u^{2}\right)\left(1-m u^{2}\right)\right]^{-1 / 2}
$$

The existence of a spacetime point $\left(t^{*}, r^{*}\right)$ where $\chi(t, r)$ vanishes is, in general, associated with the change of the winding number $N_{\chi}(t)$. It has been shown in Ref. [10] that the change of the winding number $N_{\chi}(t)$ plays an important role in fermion number violation (see also Ref. [18] for related results). Indeed, we have studied several LS solutions with $\epsilon<1 / 2$ and found that the necessary condition (3.25) for the existence of a fermion zero mode is never satisfied. In the following, we shall therefore only consider LS solutions with $\epsilon \geq 1 / 2$. But before we turn to the fermion zero modes, we mention one particular aspect of the LS gauge field background for $\epsilon \geq 1 / 2$.

\section{B. LS quasi-sphaleron}

For energy parameter $\epsilon \geq 1 / 2$, the field $\chi(t, r)$ has at least one zero at a particular spacetime point. In order to simplify the analysis, we take for the $\zeta$ and $\tau_{0}$ parameters in the solution (5.6b) the following values:

$$
\zeta=+1, \quad \tau_{0}=-(8 \epsilon)^{-1 / 4} K(m),
$$

with $m$ defined by Eq. (5.7). For the choice of $\tau_{0}$ from Eq. (5.11), one of the zeros of $\chi(t, r)$ occurs at the time slice $t=t_{0}=0$. Also note that for this $\tau_{0}$ the LS solution has time-reversal (anti)symmetry, namely $\rho(-t, r)=\rho(t, r)$ and $\mathcal{R}(-t, r)=-\mathcal{R}(t, r)$, with $\rho(t, r)$ and $\mathcal{R}(t, r)$ defined in Eq. (3.14). In the following, we consider the $t=0$ time slice of these particular LS background gauge fields [parameters $\zeta=+1, \tau_{0}$ from Eq. (5.11), and $\epsilon \geq 1 / 2$ ], for which the zero of $\chi(0, r)$ occurs at $r=1$, according to Eq. (5.8).

The LS gauge field background at $t=0$ is represented by

$$
a_{\mu}(0, r)=0, \quad \chi(0, r)=\sin w(0, r)
$$


up to a $U(1)$ gauge transformation (2.11). Given that the real function $\chi(0, r)$ vanishes and changes sign at $r=1$, the configuration of Eq. (5.12) qualitatively resembles the sphaleron solution of the electroweak $S U(2)$ Yang-Mills-Higgs theory [6, 17]. For this reason, we call the configuration given by Eq. (5.12) the "LS quasi-sphaleron." Note that the LS quasisphaleron does not satisfy the static field equations, since the energy changes under a scale transformation of the fields (there is no natural mass scale for classical Yang-Mills theory).

We will now show that this LS quasi-sphaleron corresponds to the top of a potential energy barrier which separates configurations with different $N_{\chi}$, just like the electroweak sphaleron [6]. The energy functional [7, 10] for $(1+1)$-dimensional gauge field solutions can, in fact, be written as

$$
E=E_{K}(t)+E_{P}(t)
$$

with

$$
\begin{aligned}
& E_{K}(t)=\frac{8 \pi}{g^{2}} \int_{0}^{\infty} \mathrm{d} r\left[\frac{1}{8 \rho^{2}}\left(\partial_{t} \rho^{2}\right)^{2}+\frac{1}{2 \rho^{2}}\left(\partial_{t} \psi\right)^{2}+\frac{1}{2 \rho^{2}}\left(\partial_{r} \psi\right)^{2}+\frac{\psi^{2}}{r^{2}}\right], \\
& E_{P}(t)=\frac{8 \pi}{g^{2}} \int_{0}^{\infty} \mathrm{d} r\left[\frac{1}{8 \rho^{2}}\left(\partial_{r} \rho^{2}\right)^{2}+\frac{\left(\rho^{2}-1\right)^{2}}{4 r^{2}}\right]
\end{aligned}
$$

where $\rho(t, r)$ equals $|\chi(t, r)|$ and $\psi(t, r)$ is the (bosonic) gauge-invariant field defined by

$$
-2 \epsilon_{\mu \nu} \psi(t, r) \equiv r^{2} f_{\mu \nu}(t, r)
$$

Here, we have divided the energy into the kinetic part $E_{K}$ and potential part $E_{P}$. The reason for putting the $\psi$-dependent terms into the kinetic part of the energy is that, for the gauge choice $a_{0}=0$, the scalar field $\psi$ becomes proportional to the time derivative of the $a_{1}$ field, namely $\psi=-r^{2} \partial_{0} a_{1}$.

Consider then the potential energy $E_{P}(t)$ associated with our particular LS gauge field solutions. Using the basic properties [20] of the Jacobi elliptic function $\mathrm{cn}[u \mid m]$, one can prove that the potential energy $E_{P}(t)$ has a local maximum at $t=0$, that is, for the LS quasi-sphaleron configuration. Furthermore, the conserved total energy of the LS solution with $\tau_{0}$ parameter (5.11) is given by

$$
E=2 \epsilon E_{\text {quasi-sph }}
$$

in terms of the energy parameter $\epsilon$ and the static energy of the LS quasi-sphaleron, $E_{\text {quasi-sph }}$ $\equiv E_{P}(0)$.

Figure 1 shows the time development of $E_{P}(t)$ for the LS background with $\epsilon=1, \zeta=+1$, and $\tau_{0} \approx-1.4271$ from Eq. (5.11). The corresponding topological charge $Q \approx-0.70$ is noninteger, see Ref. [7] for further details. More importantly, the potential barrier of Fig. [1 separates two regions with different winding number $\left(\Delta N_{\chi}=-1\right)$. The LS quasi-sphaleron at $t=0$ resembles in this respect also the sphaleron of the electroweak Standard Model [6].

For comparison, consider the LS gauge field background given by the trivial solution $q=-1$ of Eq. (5.4), with the (1+1)-dimensional field configurations

$$
\psi(t, r)=0, \quad \chi(t, r)=\sin w(t, r),
$$


as follows from Eqs. (2.11), (5.2), and (5.15). This corresponds to the de Alfaro, Fubini and Furlan (AFF) solution [22]. Note that the AFF gauge field coincides with the LS quasisphaleron (5.12) at $t=0$. On the other hand, the AFF solution has complete time-reversal symmetry and the kinetic energy $E_{K}(t)$ as given by Eq. (5.14a) is zero at $t=0$ (see Fig. 2). This result suggests that the AFF solution provides the time-dependent gauge field solution with minimum total energy to form the LS quasi-sphaleron. In other words, the AFF gauge field simulates an "imploding and exploding LS quasi-sphaleron;" cf. Ref. [23]. (For the electroweak sphaleron, the dynamics of the gauge and Higgs fields has been studied numerically. See, for example, Refs. [24,25].)

\section{Fermion zero mode of the LS quasi-sphaleron}

We now turn to the fermion zero-eigenvalue equation (3.9) for the $t=0$ LS gauge field of the previous subsection, i.e. the LS quasi-sphaleron (5.12). Note that the gauge-invariant function $\lambda(0, r) \equiv \rho(0, r) / r$ is nondifferentiable at $r=1$, which is the only point where $\rho(0, r) \equiv|\chi(0, r)|$ vanishes. For this reason, we introduce a differentiable field $\tilde{\lambda}(0, r)$, defined by

$$
\begin{aligned}
\tilde{\lambda}(0, r) & \equiv \kappa(0, r) / r \\
\kappa(0, r) & \equiv \operatorname{sgn}(1-r)|\chi(0, r)| .
\end{aligned}
$$

Then, $\chi(0, r)$ can be represented by

$$
\chi(0, r)=\kappa(0, r) \exp [i \tilde{\varphi}(0, r)],
$$

where $\tilde{\varphi}(0, r)$ is a differentiable function of $r$.

It is a simple exercise to verify that the LS field $\kappa(0, r)$ has the following inversion symmetry:

$$
\kappa(0,1 / r)=-\kappa(0, r) .
$$

This inversion symmetry, most likely, traces back to the conformal symmetry transformation $x^{\mu} \rightarrow x^{\mu} / x^{2}$ of classical Yang-Mills theory. Without loss of generality, we consider, in the following, smooth background fields with $\kappa(0,0)=1$ and $\tilde{\varphi}(0,0)=0$.

In terms of the differentiable fields $\tilde{\lambda}$ and $\tilde{\mathcal{R}} \equiv\left(a_{1}-\partial_{r} \tilde{\varphi}\right) / 2$, one finds that the zero-energy fermion equations (3.13) at $t=0$ become

$$
\begin{aligned}
\partial_{r} \Theta & =-\tilde{\lambda} \sin 2 \Theta+\tilde{\mathcal{R}}, \\
\partial_{r}|\Psi| & =\tilde{\lambda}|\Psi| \cos 2 \Theta,
\end{aligned}
$$

with boundary conditions

$$
\Theta(0,0)=|\Psi(0,0)|=0 .
$$

Using the inversion symmetry (5.20) and the asymptotics (3.11) of the rotator, it follows from Eq. (5.21b) that there exists a fermion zero mode if and only if $\lim _{r \rightarrow \infty} \Theta(0, r)=N \pi$, with $N$ an integer. 
We can now explicitly construct the fermion zero mode for the LS background at $t=0$. Using the field equations [7, 10], it is relatively straightforward to show that

$$
\left.\tilde{\mathcal{R}}(0, r) \propto \frac{\mathrm{d}^{2} q}{\mathrm{~d} \tau^{2}}\right|_{\tau=0} \propto \operatorname{cn}[K(m) \mid m]=0,
$$

where the last identity can be found, for example, in Ref. [20]. [Note that vanishing $\tilde{\mathcal{R}}$ does not contradict the necessary condition (3.25) that was derived for Eqs. (3.13) with a function $\lambda \geq 0$, whereas Eqs. (5.21) have a function $\tilde{\lambda}$ that changes sign.]

For $\tilde{\mathcal{R}}=0$, the solutions to Eqs. (5.21) are simply given by

$$
\begin{aligned}
\Theta(0, r) & =0 \\
|\Psi(0, r)| & =\left|\Psi\left(0, r_{0}\right)\right| \exp \left[\int_{r_{0}}^{r} \mathrm{~d} r^{\prime} \tilde{\lambda}\left(0, r^{\prime}\right)\right] .
\end{aligned}
$$

The argument following Eq. (5.22) ensures that the solution represented by Eqs. (5.24ab) is normalizable, which completes the construction of the fermion zero mode. The inversion symmetry (5.20), together with the result (5.23), provides a sufficient condition for the existence of the fermion zero mode.

For $\epsilon \geq 1 / 2$, the fermion zero mode amplitude $|\Psi(0, r)|$ of Eq. (5.24b) is shown in Fig. 3, with an arbitrary normalization. Specifically, the $(3+1)$-dimensional fermion zero mode is purely left-handed and given by Eqs. (2.6) and (2.9), with the two-component spinor $\Psi_{l}=\Psi$ from Eqs. (3.7), (3.12), and (5.24). These last equations are to be evaluated with the functions $\kappa(0, r)$ and $\tilde{\varphi}(0, r)$ defined in Eq. (5.19). [Of course, the solution can also be

obtained from Eqs. (3.13), which are given in terms of $\lambda(0, r)$ and $\varphi(0, r)$. In this case, the function $\Theta(0, r)$ is found to have a step function at $r=1$, but so does the transformation matrix $\Lambda$ from Eq. (3.8).]

To summarize, the LS quasi-sphaleron at $t=0$ has a chiral fermion zero mode and resembles in this respect the electroweak sphaleron which also has a chiral fermion zero mode (see Refs. [26 28] and references therein). The fermion zero modes of the LS quasisphaleron and the electroweak sphaleron are qualitatively the same. Moreover, there is spectral flow associated with both the electroweak sphaleron (see Refs. 27 29]) and the LS quasi-sphaleron (see Sec. V D 3 below). This behavior differs from that of the AFF solution (an "imploding and exploding LS quasi-sphaleron"), for which the fermion zero mode exists at all times 23], without level crossing.

\section{Level crossings for large energy parameter $\epsilon$}

Throughout this subsection, we consider the specific LS gauge field solution with parameters $\epsilon=20, \zeta=+1$, and $\tau_{0} \approx-0.54197$ from Eq. (5.11). Figures 1 and 5 give the behavior of the potential energy $E_{P}(t)$ of this solution. The corresponding topological charge $Q \approx-0.13$ is noninteger; cf. Ref. [7]. The LS quasi-sphaleron at $t=0$ is the same as for the $\epsilon=1$ case, but no longer corresponds to a global maximum of $E_{P}(t)$.

In order to determine the spectral flow for this particular gauge field background, all fermion zero modes need to be determined, to which we turn first. 


\section{Extra fermion zero modes from changes in winding number}

According to Eq. (5.8), there are zeros of this LS field $\chi(t, r)$ at the spacetime points

$$
\begin{aligned}
& \left(t_{-1}, r_{-1}\right) \approx(-1.889,2.137), \quad\left(t_{0}, r_{0}\right)=(0,1), \\
& \left(t_{+1}, r_{+1}\right) \approx(+1.889,2.137) .
\end{aligned}
$$

The winding number $N_{\chi}(t)$ takes the following values in the different time regions:

$$
N_{\chi}(t)= \begin{cases}-1 & \text { for } t \in\left(-\infty, t_{-1}\right) \\ 0 & \text { for } t \in\left(t_{-1}, t_{0}\right) \\ -1 & \text { for } t \in\left(t_{0}, t_{+1}\right) \\ 0 & \text { for } t \in\left(t_{+1},+\infty\right)\end{cases}
$$

This gives the global winding factor

$$
\Delta N_{\chi}[+\infty,-\infty]=0-(-1)=1 \text {. }
$$

As discussed in Sec. V C, there exists a fermion zero mode at $t=t_{0}=0$ (see Fig. 3). But there are two more fermion zero modes precisely at $t=t_{-1}$ and $t=t_{+1}$. Three preliminary steps are necessary for the proof.

First, define smooth fields $\tilde{\lambda}\left(t_{n}, r\right)$ and $\tilde{\mathcal{R}}\left(t_{n}, r\right)$ at the time slices $t=t_{n}$, for $n= \pm 1$,

$$
\begin{aligned}
\tilde{\lambda}\left(t_{n}, r\right) & \equiv \kappa\left(t_{n}, r\right) / r, \\
\tilde{\mathcal{R}}\left(t_{n}, r\right) & \equiv\left[a_{1}\left(t_{n}, r\right)-\partial_{r} \tilde{\varphi}\left(t_{n}, r\right)\right] / 2,
\end{aligned}
$$

with the differentiable function $\kappa\left(t_{n}, r\right) \equiv \rho\left(t_{n}, r\right) \times \operatorname{sgn}\left(r_{n}-r\right)$ and the smooth argument $\tilde{\varphi}\left(t_{n}, r\right)$ of the Higgs-like field $\chi=\kappa \exp [i \tilde{\varphi}]$.

Second, perform a scale transformation $x=r / r_{n}$, so that the radial point $r=r_{n}$ where the field $\chi\left(t_{n}, r\right)$ vanishes corresponds to $x=1$.

Third, establish that the Lüscher-Schechter solution for the parameters chosen has the following inversion symmetry at fixed time slices $t=t_{n}$ :

$$
\begin{aligned}
\tilde{\lambda}\left(t_{n}, 1 / x\right) & =-x^{2} \tilde{\lambda}\left(t_{n}, x\right), \\
\tilde{\mathcal{R}}\left(t_{n}, 1 / x\right) & =-x^{2} \tilde{\mathcal{R}}\left(t_{n}, x\right),
\end{aligned}
$$

for $n= \pm 1$. The existence of this inversion symmetry has been verified analytically with the help of Mathematica 4.0 [21].

After these preliminaries, we turn to the possible existence of fermion zero modes at the time slices $t=t_{ \pm 1}$. Consider the zero-energy fermion equations given by Eqs. (3.13) at $t=t_{n}$, for $n= \pm 1$, with the smooth background fields $\tilde{\lambda}\left(t_{n}, r\right)$ and $\tilde{\mathcal{R}}\left(t_{n}, r\right)$. The chiral Yang-Mills theory (2.1) is scale invariant and the rescaling $x=r / r_{ \pm 1}$ at $t=t_{ \pm 1}$ does not alter the structure of Eqs. (3.13):

$$
\begin{aligned}
\frac{\mathrm{d} \Theta}{\mathrm{d} x}=-\tilde{\lambda}(x) \sin 2 \Theta(x)+\tilde{\mathcal{R}}(x), & \Theta(0)=0, \\
\frac{\mathrm{d}|\Psi|}{\mathrm{d} x}=\tilde{\lambda}(x)|\Psi(x)| \cos 2 \Theta(x), & |\Psi(0)|=0,
\end{aligned}
$$


with the dependence on $t_{ \pm 1}$ temporarily dropped. The differential equations (5.30) are symmetric under the inversion transformation $x \leftrightarrow 1 / x$ and so are their solutions $\Theta(x)$ and $|\Psi(x)|$. The inversion symmetry implies

$$
\lim _{x \rightarrow 0} \Theta=\lim _{x \rightarrow \infty} \Theta=0, \quad \lim _{x \rightarrow 0}|\Psi|=\lim _{x \rightarrow \infty}|\Psi|=0,
$$

with $|\Psi| \propto 1 / x$ for large $x$. This shows that there exist fermion zero modes at both $t=t_{-1}$ and $t=t_{+1}$.

The inversion symmetry (5.29), after the appropriate scale transformation, provides again a sufficient condition for the existence of fermion zero modes at $t=t_{ \pm 1}$. For Lüscher-Schechter gauge field backgrounds with arbitrary $\tau_{0}$ and $\epsilon \geq 1 / 2$, the inversion symmetry (5.29) holds, in fact, at any time slice $t=t_{n}$ where $\chi(t, r)$ has a zero. This then proves the existence of fermion zero modes at all $t_{n}$.

Figure 6 gives the profile functions of the fermion zero mode at $t=t_{-1}$, obtained from the numerical solution of Eqs. (5.30). The profile functions of the fermion zero mode at $t=t_{+1}$ are identical, except for a change of sign of $\Theta$. These functions are quantitatively different from those of the LS quasi-sphaleron [see Eqs. (5.24) and Fig. 3], but qualitatively the same.

\section{Extra fermion zero modes from changes in twist number}

In order to locate all possible level crossings, we are guided by the change of the spinor twist number $N_{\Theta}(t)$. The spinor twist number $N_{\Theta}(t)$ takes the following values in the different time regions:

$$
N_{\Theta}(t)= \begin{cases}+1 & \text { for } \quad t \in\left(-\infty,-t_{a}\right) \\ 0 & \text { for } t \in\left(-t_{a},+t_{a}\right) \\ -1 & \text { for } \quad t \in\left(+t_{a},+\infty\right)\end{cases}
$$

with the numerical estimate $t_{a} \approx 2.924$. The corresponding twist factor is thus given by

$$
\Delta N_{\Theta}[+\infty,-\infty]=-1-1=-2 .
$$

In addition to the fermion zero modes at $t_{0}$ and $t_{ \pm 1}$, which are associated with the change of the gauge field winding number $N_{\chi}(t)$, there exist two more fermion zero modes precisely at $t= \pm t_{a}$. The analysis of these fermion zero modes is straightforward and the normalizability condition is found to hold, provided $\Theta\left( \pm t_{a}, r\right)$ approaches a half-oddinteger multiple of $\pi$ as $r \rightarrow \infty$; see Eq. (3.13b). Note that the exact value of $t_{a}$ is defined implicitly by the relation $\lim _{R \rightarrow \infty} \Theta\left( \pm t_{a}, R\right)=\mp \pi / 2$, where the solution $\Theta\left( \pm t_{a}, R\right)$ of the differential equation (3.13a) with boundary condition (3.15a) can be obtained by the method of successive approximations 14, 15].

Figure 7 gives the profile functions of the fermion zero mode at $t=-t_{a}$, obtained from the numerical solution of Eqs. (3.13) and (3.15). The profile functions of the fermion zero

mode at $t=+t_{a}$ are identical, except for a change of sign of $\Theta$. Figures 8 and 9 show the time-variation of the solutions $\Theta(t, r)$ of the transformed Riccati equation (3.13a) around $t= \pm t_{a}$, which demonstrates that the fermion zero modes at $t= \pm t_{a}$ sit at bifurcation points 
for different $N_{\Theta}$ 's. [These results provide an example for the general discussion leading up to Eqs. (4.14) in Sec. IV A 2.] Obviously, Figs. 8 and 9 are related, because of the time-reflection properties of the background fields mentioned below Eq. (5.11).

The fermion zero modes at $t= \pm t_{a}$ are qualitatively different from the ones at $t_{0}$ and $t_{ \pm 1}$ (compare Fig. 7 with Figs. 3 and 6). These fermion zero modes occur, in fact, for Higgslike fields $\chi\left( \pm t_{a}, r\right)$ without zeros. This differs from the cases discussed in the literature 10,18,30. Apparently, the long-range behavior of the background $S U(2)$ gauge fields plays a crucial role for the existence of these extra fermion zero modes (see also the discussion in Sec. VI).

\section{Spectral flow}

For the fermion zero modes at $t=-t_{a}, t_{-1}, t_{0}, t_{+1}$, and $+t_{a}$, we calculate the following time-gradients of the energy eigenvalue of the effective Dirac Hamiltonian:

$$
\begin{aligned}
& \left.\frac{\mathrm{d} E}{\mathrm{~d} t}\right|_{t=-t_{a}}=\left.\frac{\mathrm{d} E}{\mathrm{~d} t}\right|_{t=+t_{a}} \approx-0.03<0, \\
& \left.\frac{\mathrm{d} E}{\mathrm{~d} t}\right|_{t=t_{-1}}=\left.\frac{\mathrm{d} E}{\mathrm{~d} t}\right|_{t=t_{+1}} \approx+0.08>0, \\
& \left.\frac{\mathrm{d} E}{\mathrm{~d} t}\right|_{t=t_{0}} \approx-5.00<0 .
\end{aligned}
$$

In addition, we have checked that there are no further fermion zero modes, at least for the time interval $[-200,+200]$.

The level crossings corresponding to Eq. (5.34) give the following value for the spectral flow (starting from $t=-t_{a}$ and ending at $t=+t_{a}$ ):

$$
\mathcal{F}[+\infty,-\infty]=-1+1-1+1-1=-1 .
$$

It would certainly be interesting to calculate the spectrum of the time-dependent effective Dirac Hamiltonian numerically (cf. Ref. [24]) in order to see whether or not the pattern (5.35) corresponds to a single energy level crossing $E=0$ five times. Anyway, the total value (5.35) agrees with the spectral flow obtained from the relation (4.39):

$$
\begin{aligned}
\mathcal{F}[+\infty,-\infty] & =\Delta N_{\chi}[+\infty,-\infty]+\Delta N_{\Theta}[+\infty,-\infty] \\
& =1-2=-1,
\end{aligned}
$$

where the results (5.27) and (5.33) have been used. This demonstrates the role of the twist factor $\Delta N_{\Theta}$ for the spectral flow, which has not been noticed before to our knowledge.

Recall that the explicit results of the present subsection are for the particular LS gauge field background with energy parameter $\epsilon=20$, together with $\zeta$ and $\tau_{0}$ from Eq. (5.11). The spectral flow for LS background gauge fields turns out to be solely given by the winding factor $\Delta N_{\chi}$ if $\epsilon$ is smaller than $\epsilon^{*} \approx 5.37071$, which is the numerical solution of the following equation: 


$$
\epsilon^{*}=\frac{1}{8}\left[\frac{4}{\pi} K\left(\frac{1+\sqrt{2 \epsilon^{*}}}{2 \sqrt{2 \epsilon^{*}}}\right)\right]^{4} .
$$

Briefly, the argument runs as follows. First, the numerical results (and a heuristic argument) give $t_{a} \geq t_{+1}$. Second, the times $t_{ \pm 1}$ move toward $\pm \infty$ as $\epsilon$ approaches $\epsilon^{*}$ from above; see Eqs. (5.8) and (5.11). Together, this implies that the contribution of the twist factor to the spectral flow vanishes for $\epsilon \leq \epsilon^{*}$, giving $\mathcal{F}[+\infty,-\infty]=\Delta N_{\chi}[+\infty,-\infty]$. (The winding factor $\Delta N_{\chi}[+\infty,-\infty]$ is, of course, identically zero for $\epsilon<1 / 2$.)

To summarize, the twist factor $\Delta N_{\Theta}$ can play a significant role for the spectral flow in certain LS gauge field backgrounds, provided the energy parameter is large enough $\left(\epsilon>\epsilon^{*}\right)$.

\section{DISCUSSION}

In this paper, we have studied real-time anomalous fermion number violation by directly investigating the zero-eigenvalue equation (3.1a) of the time-dependent effective Dirac Hamiltonian for spherically symmetric massless chiral fermions and $S U(2)$ Yang-Mills gauge field backgrounds. For these spherically symmetric $S U(2)$ gauge field backgrounds, we have found a relation between the spectral flow and two characteristics of the gauge fields. Physics applications of this result are based on the assumption that anomalous production of fermions is confined to the spherically symmetric partial wave; cf. Ref. [17]. Perhaps the most important application would be for electroweak baryon number violation in the early universe, in particular at temperatures above the electroweak phase transition (see Ref. 31 for a review).

Since we adopt an approach different from the one of previous work [5, 9, 10], we are able to observe certain new features of real-time fermion number violation within the spherically symmetric Ansatz. These features include the spinor twist number $N_{\Theta}(t)$ obtained from the Riccati equation (3.16) at a single fixed time $t$ and the corresponding twist factor $\Delta N_{\Theta}\left[t_{f}, t_{i}\right]$ which is the change of the spinor twist number over the time interval $\left[t_{i}, t_{f}\right]$, with $t_{i}<t_{f}$. Relation (4.39) then gives the spectral flow $\mathcal{F}$ as the sum of this twist factor $\Delta N_{\Theta}$ and the winding factor $\Delta N_{\chi}$, which is the change of the Chern-Simons number $N_{\chi}$ of the associated vacuum sectors of the gauge field background. Mathematically, the relation (4.39) takes the form of an index theorem, restricted to spherically symmetric fields; cf. Ref. [5].

In order to get better insight into the meaning of this relation (4.39), we have investigated level crossings for a particular class of Lüscher-Schechter (LS) gauge field solutions [12, 13. The results clearly demonstrate the role of the twist factor for the spectral flow. See, in particular, Eqs. (5.35) and (5.36). The nonvanishing global effect of the twist factor on the spectral flow is partly due to the fact that the LS gauge fields form propagating solitons in the effective (1+1)-dimensional theory; cf. Refs. [7, 10]. The fields are thus nondissipative in the $(1+1)$-dimensional world. For such background gauge fields, the field-theoretic approach adopted in Refs. [9:10] is, strictly speaking, not applicable.

Although nondissipative in the $(1+1)$-dimensional context, these LS gauge field solutions are dissipative in $(3+1)$-dimensional spacetime. The $(3+1)$-dimensional energy density, which is obtained from the (1+1)-dimensional energy density (5.14) by dividing by $4 \pi r^{2}$, approaches zero uniformly for early and late times $(t \rightarrow \pm \infty)$. 
At this moment, we propose to classify dissipative spherically symmetric $S U(2)$ gauge field solutions into two categories. A spherically symmetric $S U(2)$ gauge field solution is called strongly dissipative, if both the $(3+1)$-dimensional and $(1+1)$-dimensional energy densities approach zero uniformly for large times $(t \rightarrow \pm \infty)$. On the other hand, a spherically symmetric $S U(2)$ gauge field solution is called weakly dissipative, if the $(3+1)$-dimensional energy density dissipates with time, but not the $(1+1)$-dimensional energy density. Note that the LS gauge field solutions considered in Sec. V D are weakly dissipative, according to this terminology.

For strongly dissipative spherically symmetric $S U(2)$ gauge field solutions, the rotator $\mathcal{R}(t, r)$ in the transformed Riccati equation (3.13a) lacks the strength to give a nonzero spinor twist number $N_{\Theta}$ at large times (see Appendix A for the proof). The relation (4.39) then predicts that the spectral flow $\mathcal{F}$ is solely given by the winding factor $\Delta N_{\chi}$, which reproduces the known result [9,10]. An isolated change of spinor twist number can still contribute to the local pattern of level crossing (4.32).

For weakly dissipative or nondissipative spherically symmetric $S U(2)$ gauge field solutions, a nonvanishing twist factor $\Delta N_{\Theta}$ can even make a global contribution to the spectral flow $\mathcal{F}$. As mentioned above, this has been verified for certain LS solutions [12,13]. This behavior does not follow directly from the perturbative triangle anomaly [1] [3], which detects only the (noninteger) topological charge. Recall that the standard perturbative calculations (Feynman diagrams) essentially neglect the interactions of incoming and outgoing particles, i.e. the interactions are "turned off" in the asymptotic regions [32].

To summarize, the spectral flow result (4.39) does not assume strongly dissipative spherically symmetric $S U(2)$ Yang-Mills gauge field backgrounds, in contrast to previous studies [5. 9, 10]. It may, therefore, be applied to real-time anomalous fermion number violation for weakly dissipative or nondissipative spherically symmetric $S U(2)$ Yang-Mills gauge field backgrounds. Moreover, preliminary results indicate that the relation (4.39) can be adapted to the case of chiral fermions interacting with spherically symmetric $S U(2)$ Yang-Mills and Higgs fields.

The main outstanding problem is, of course, to understand the role of the (appropriately generalized) twist factor in the full $(3+1)$-dimensional $S U(2)$ Yang-Mills theory, not just the subspace of spherically symmetric configurations. Also, the corresponding index theorem needs to be established for the long-range Yang-Mills gauge fields considered; cf. Ref. [30] and references therein. Finally, the proper definition (if at all possible) of the second-quantized fermion number operator in general nondissipative Yang-Mills gauge field backgrounds requires further study.

\section{APPENDIX A: SPINOR TWIST NUMBER AND STRONGLY DISSIPATIVE}

\section{$S U(2)$ GAUGE FIELDS}

In this appendix, we calculate the asymptotic spinor twist number $N_{\Theta}(t)$, defined by Eqs. (3.13a), (3.15a), and (3.20) in the main text, for strongly dissipative spherically symmetric $S U(2)$ gauge field solutions.

From the (1+1)-dimensional energy density (5.14) and the corresponding field equations (see, in particular Eqs. (3.8) and (3.10) of Ref. [10]), the condition of strong dissipation 
implies that there exists a small positive quantity $\epsilon(t) \ll 1$ at large $|t|$, so that

$$
\left|r^{-1}-\lambda(t, r)\right|<\epsilon(t) \quad \text { and } \quad|\mathcal{R}(t, r)|<\epsilon(t)
$$

for $0 \leq r<\infty$, together with the limit

$$
\lim _{|t| \rightarrow \infty} \epsilon(t)=0
$$

See Eqs. (2.9), (2.15), and (3.14) in the main text for the definition of $\lambda(t, r)$ and $\mathcal{R}(t, r)$.

From the bounds (A1), we immediately obtain

$$
0<r^{-1}-2 \epsilon(t)<\lambda(t, r)-|\mathcal{R}(t, r)|
$$

for $0 \leq r<R_{\epsilon} \equiv[2 \epsilon(t)]^{-1}$. Since regular finite-energy gauge fields obey $\lim _{r \rightarrow \infty} \mathcal{R} / \lambda=0$ [see Eq. (3.11) in the main text], we have from Eq. (A3) the following inequality for arbitrary $r$ :

$$
0 \leq|\mathcal{R}(t, r)|<\lambda(t, r)
$$

provided $|t|$ is sufficiently large.

With the inequality (A4) in hand, we are able to establish that the spinor twist number $N_{\Theta}(t)$ vanishes asymptotically. This can be shown by contradiction. Consider the transformed Riccati equation,

$$
\begin{aligned}
\partial_{r} \Theta(t, r) & =\mathcal{D}(t, r)+\mathcal{R}(t, r), \quad \Theta(t, 0)=0, \\
\mathcal{D}(t, r) & \equiv-\lambda(t, r) \sin 2 \Theta(t, r)
\end{aligned}
$$

at sufficiently large $|t|$. Now, assume that there exists a time slice $t=\bar{t}$ where the background fields obey inequality (A4) and that the solution $\Theta(\bar{t}, r)$ of Eq. (A5) belongs to the class $S_{N}(\bar{t})$, with positive integer $N$ (the case of negative $N$ will be dealt with later). See Eq. (3.19a) in the main text for the definition of the solution class $S_{N}$.

Strong dissipation gives $|\chi(\bar{t}, r)| \neq 0$, so that the background fields $\lambda(\bar{t}, r)$ and $\mathcal{R}(\bar{t}, r)$ are smooth. This implies that the solution $\Theta(\bar{t}, r) \in S_{N}(\bar{t})$, with $N \geq 1$, is continuous and must cross the value $+\pi / 4$ at least once. Define $r_{+}$to be the largest radial distance for which $\Theta(\bar{t}, r)=+\pi / 4$. In order for $\Theta(\bar{t}, r)$ to reach the asymptotic value $N \pi$, the slope of $\Theta(\bar{t}, r)$ at $r=r_{+}$clearly must be nonnegative,

$$
\partial_{r} \Theta\left(\bar{t}, r_{+}\right) \geq 0
$$

as long as $\Theta \in S_{N}(\bar{t})$, with $N \geq 1$.

On the other hand, Eqs. (A4) and (A5), together with the fact that $\sin 2 \Theta\left(\bar{t}, r_{+}\right)=+1$, give the following inequality:

$$
\partial_{r} \Theta\left(\bar{t}, r_{+}\right)=-\lambda\left(\bar{t}, r_{+}\right)+\mathcal{R}\left(\bar{t}, r_{+}\right)<0
$$

This last result contradicts the earlier result (A6), which was based on the assumption that $\Theta(\bar{t}, r) \in S_{N}(\bar{t})$, with $N \geq 1$. Hence, $\Theta(\bar{t}, r) \notin S_{N}(\bar{t})$, for positive integer $N$. The case of 
negative integer $N$ is ruled out in the same way. The conclusion is thus that $\Theta(\bar{t}, r)$ belongs to $S_{0}(\bar{t})$.

For strongly dissipative spherically symmetric $S U(2)$ gauge field solutions, we find that the rotator $\mathcal{R}(t, r)$ at large times lacks the strength to overcome the resistance of the deviator $\mathcal{D}(t, r)$, so as to give a nonzero spinor twist number at large times. In short, we have

$$
\lim _{t \rightarrow-\infty} N_{\Theta}(t)=\lim _{t \rightarrow+\infty} N_{\Theta}(t)=0 .
$$

This result shows that the twist factor $\Delta N_{\Theta}[+\infty,-\infty] \equiv N_{\Theta}(+\infty)-N_{\Theta}(-\infty)=0$ does not contribute to the spectral flow (4.39), at least for the case of strongly dissipative spherically symmetric $S U(2)$ gauge field solutions. 


\section{REFERENCES}

[1] S.L. Adler, Phys. Rev. 177, 2426 (1969).

[2] J. Bell and R. Jackiw, Nuovo Cimento A 51, 47 (1969).

[3] G. 't Hooft, Phys. Rev. Lett. 37, 8 (1976).

[4] A. Belavin, A. Polyakov, A. Schwarz, and Yu. Tyupkin, Phys. Lett. B59, 85 (1975).

[5] N.H. Christ, Phys. Rev. D21, 1591 (1980).

[6] F.R. Klinkhamer and N.S. Manton, Phys. Rev. D30, 2212 (1984).

[7] E. Farhi, V.V. Khoze, and R. Singleton, Phys. Rev. D47, 5551 (1993).

[8] E. Farhi, J. Goldstone, S. Gutmann, K. Rajagopal, and R. Singleton, Phys. Rev. D51, 4561 (1995).

[9] T.M. Gould and S.D.H. Hsu, Nucl. Phys. B446, 35 (1995).

[10] V.V. Khoze, Nucl. Phys. B445, 270 (1995).

[11] E. Witten, Phys. Rev. Lett. 38, 121 (1977).

[12] M. Lüscher, Phys. Lett. B70, 321 (1977).

[13] B. Schechter, Phys. Rev. D16, 3015 (1977).

[14] E.L. Ince, Ordinary Differential Equations (Dover, New York, 1956).

[15] E. Hille, Lectures on Ordinary Differential Equations (Addison-Wesley, Reading, 1969).

[16] E. Witten, Phys. Lett. B117, 324 (1982).

[17] L. Yaffe, Phys. Rev. D40, 3463 (1989).

[18] Z. Guralnik, Phys. Rev. D49, 4873 (1994).

[19] F. Verhulst, Nonlinear Differential Equations and Dynamical Systems, 2nd ed. (Springer, Berlin, 1996).

[20] P. Morse and H. Feshbach, Methods of Theoretical Physics (McGraw-Hill, New York, 1953).

[21] S. Wolfram, Mathematica, 4th ed. (Cambridge University Press, Cambridge, 1999).

[22] V. de Alfaro, S. Fubini, and G. Furlan, Phys. Lett. B65, 163 (1976) .

[23] G. Gibbons and A. Steif, Phys. Lett. B346, 255 (1995).

[24] J. Ambjorn, T. Askgaard, H. Porter, and M.E. Shaposhnikov, Nucl. Phys. B353, 346 (1991); J. Ambjorn and K. Farakos, Phys. Lett. B294, 248 (1992); J. Ambjorn, K. Farakos, S. Hands, G. Koutsoumbas, and G. Thorleifsson, Nucl. Phys. B425, 39 (1994).

[25] C. Rebbi and R. Singleton, Phys. Rev. D54, 1020 (1996). 
[26] C.R. Nohl, Phys. Rev. D12, 1840 (1975).

[27] F.R. Klinkhamer and N.S. Manton, in Proceedings of the 1984 Summer Study on the Design and Utilization of the Superconducting Super Collider, edited by R. Donaldson and J. Morfin (American Physical Society, New York, 1984), p. 805.

[28] A. Ringwald, Phys. Lett. B213, 61 (1988).

[29] J. Kunz and Y. Brihaye, Phys. Lett. B304, 141 (1993).

[30] E.J. Weinberg, Phys. Rev. D24, 2669 (1981).

[31] V.A. Rubakov and M.E. Shaposhnikov, Phys. Usp. 39, 461 (1996).

[32] R.P. Feynman, Phys. Rev. 76, 769 (1949). 


\section{FIGURES}

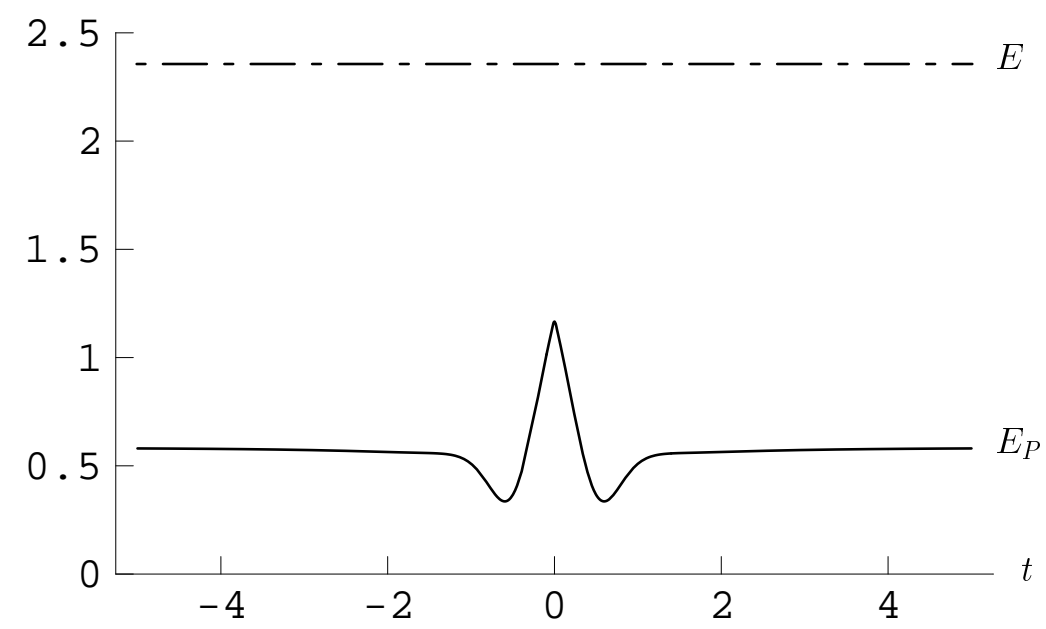

FIG. 1. Time-development of the total energy $E$ and potential energy $E_{P}(t)$ for the Lüscher-Schechter (LS) gauge field solution (2.4), (5.2), (5.6b) with parameters $\epsilon=1, \zeta=+1$, and $\tau_{0} \approx-1.4271$ from Eq. (5.11). The potential energy $E_{P}(t)$, given by Eq. (5.14b), is differentiable for all times and has a global maximum at $t=0$. The configuration at $t=0$ is called the LS quasi-sphaleron. 


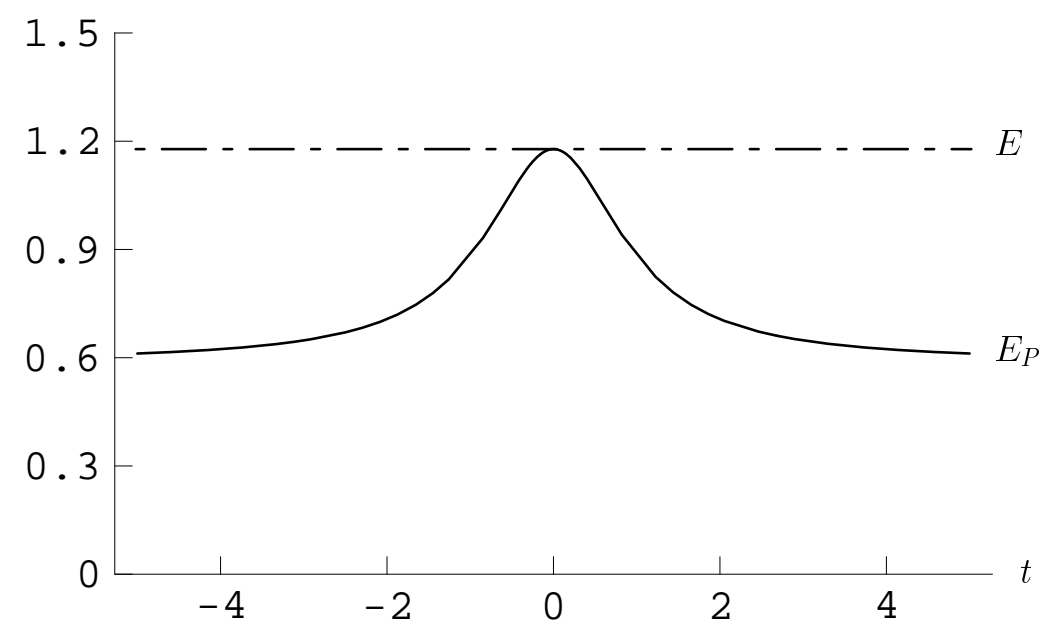

FIG. 2. Time-development of the total energy $E$ and potential energy $E_{P}(t)$ for the de Alfaro, Fubini and Furlan (AFF) gauge field solution (2.4), (5.2), (5.17). The AFF configuration at $t=0$ coincides with the LS quasi-sphaleron at $t=0$ in Fig. 1 . 


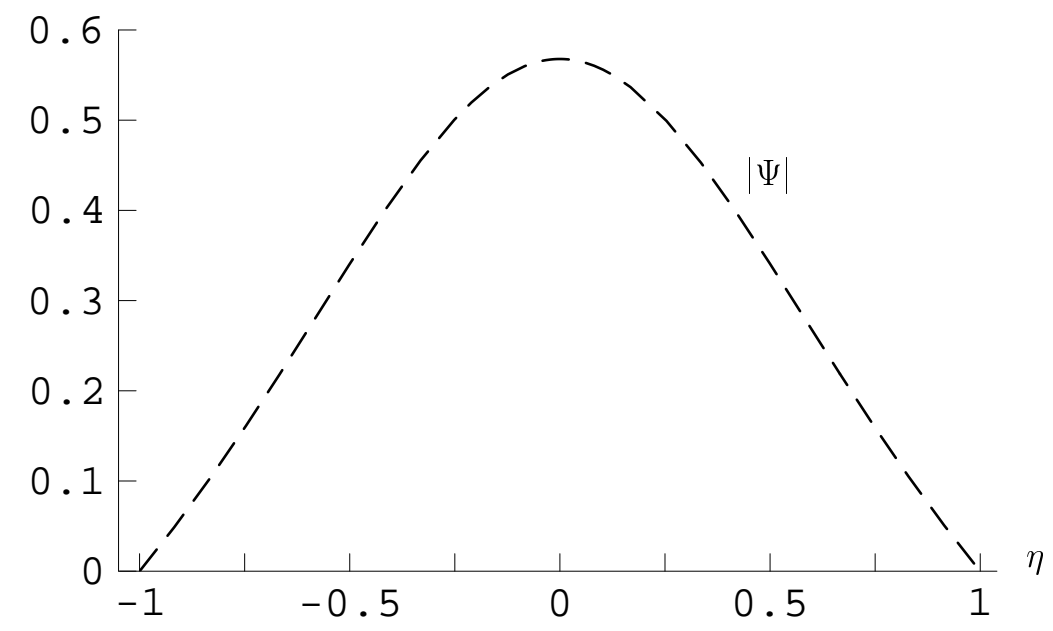

FIG. 3. Profile function $|\Psi(0, r)|$ of the fermion zero mode (5.24b) of the LS quasi-sphaleron, which corresponds to the $t=0$ configuration of Fig. 11. The dashed curve gives $|\Psi(0, r)|$ with an arbitrary normalization. The inversion symmetry $r \rightarrow 1 / r$ is made manifest by use of the compact radial coordinate $\eta \equiv(r-1) /(r+1)$. 


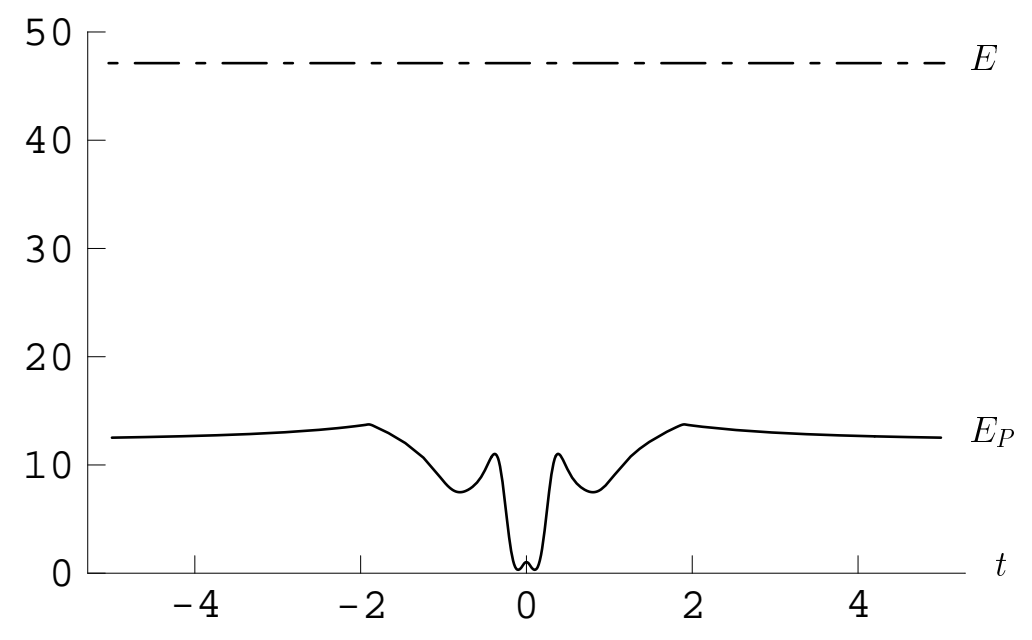

FIG. 4. Time-development of the total energy $E$ and potential energy $E_{P}(t)$ for the Lüscher-Schechter gauge field solution (2.4), (5.2), (5.6b) with parameters $\epsilon=20, \zeta=+1$, and $\tau_{0} \approx-0.54197$ from Eq. (5.11). See Fig. 5 for a close-up of $E_{P}(t)$ near $t=0$. 


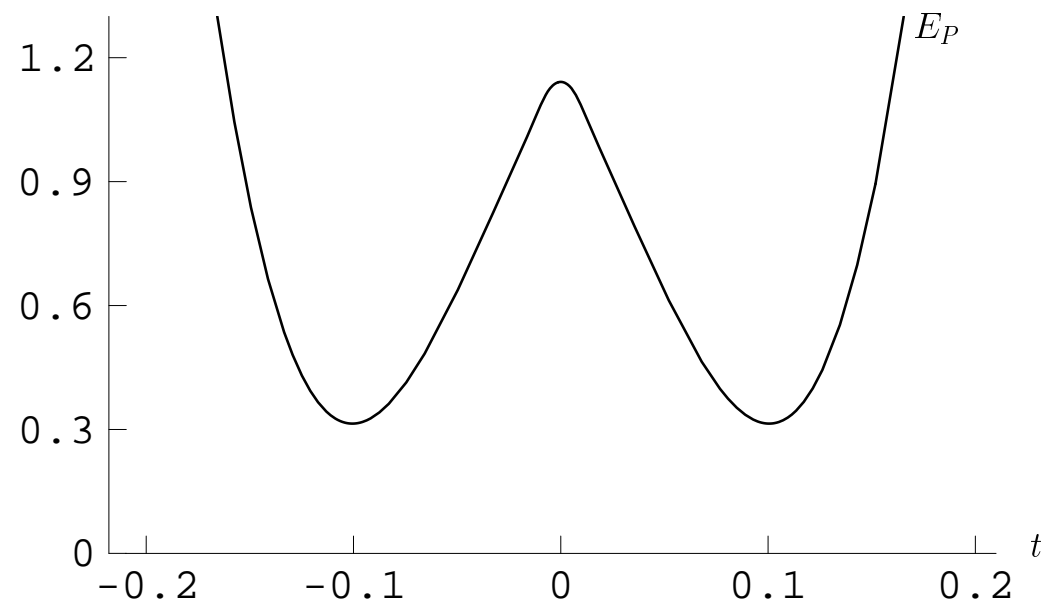

FIG. 5. Same as Fig. \$. Close-up of the potential energy $E_{P}(t)$ near $t=0$. The LS quasi-sphaleron at $t=0$ is only a local maximum of $E_{P}(t)$, unlike the case of Fig. 1 . 


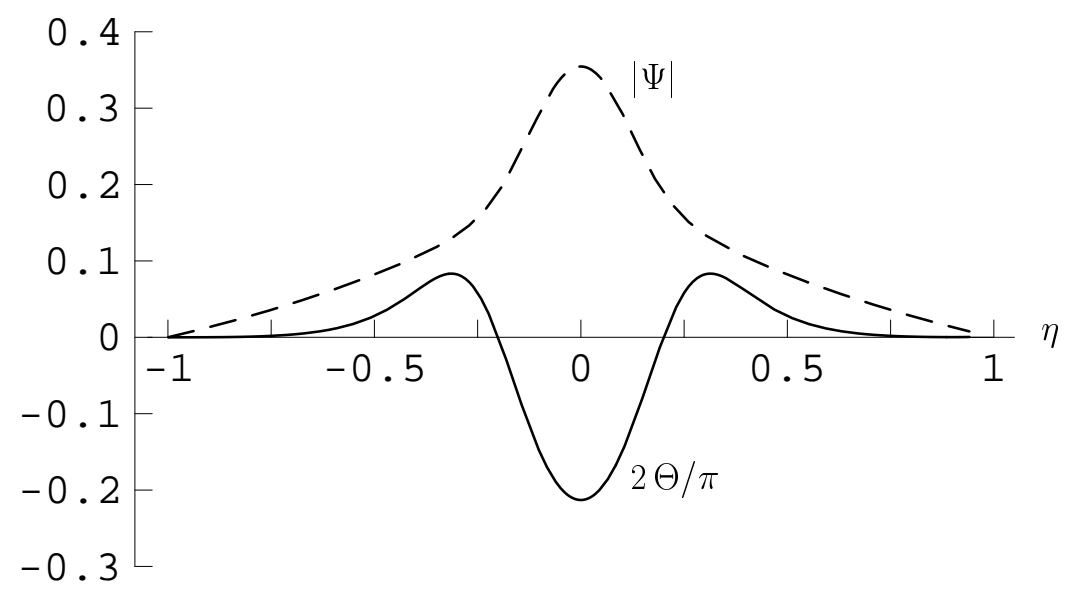

FIG. 6. Numerical solutions for the profile functions $\Theta\left(t_{-1}, r\right)$ and $\left|\Psi\left(t_{-1}, r\right)\right|$ of the fermion zero mode at $t=t_{-1} \approx-1.889$ for the Lüscher-Schechter background gauge field (2.4), (5.2), (5.6b) with parameters $\epsilon=20, \zeta=+1$, and $\tau_{0} \approx-0.54197$. The solid curve corresponds to $\Theta\left(t_{-1}, r\right) \times 2 / \pi$ and the dashed curve to $\left|\Psi\left(t_{-1}, r\right)\right|$ with an arbitrary normalization. The inversion symmetry $x \rightarrow 1 / x$, with $x \equiv r / r_{-1}$, is made manifest by use of the compact radial coordinate $\eta \equiv(x-1) /(x+1)$. 


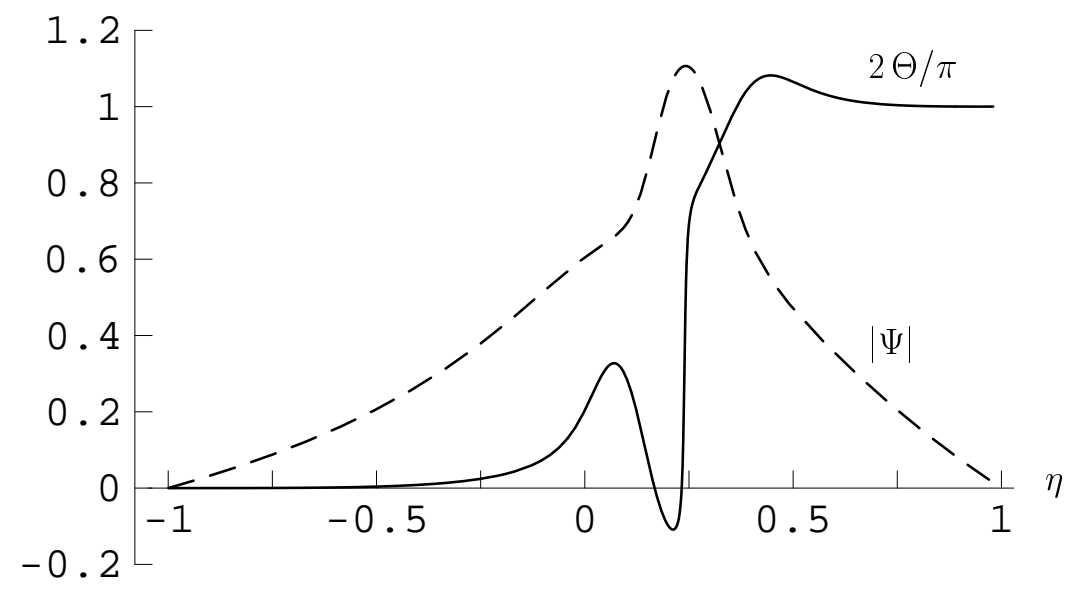

FIG. 7. Same as Fig. 6, but for the fermion zero mode at $t=-t_{a} \approx-2.924$. The solid curve corresponds to $\Theta\left(-t_{a}, r\right) \times 2 / \pi$ and the dashed curve to $\left|\Psi\left(-t_{a}, r\right)\right|$ with an arbitrary normalization. Both functions are plotted against the compact radial coordinate $\eta \equiv(x-1) /(x+1)$, with $x \equiv r / 2$. 


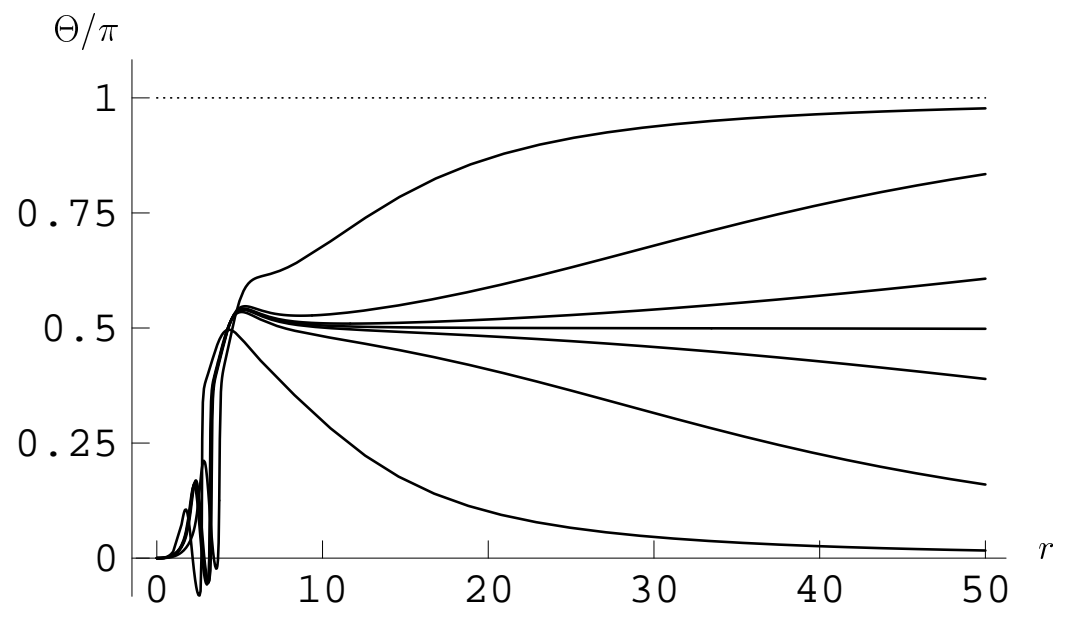

FIG. 8. Numerical solutions $\Theta(t, r)$ of the transformed Riccati equation (3.13a), with boundary condition $\Theta(t, 0)=0$, at different times around $t=-t_{a} \approx-2.924$ for the Lüscher-Schechter background gauge field (2.4), (5.2), (5.6b) with parameters $\epsilon=20, \zeta=+1$, and $\tau_{0} \approx-0.54197$. For $t=-t_{a}$, there is a normalizable fermion zero mode, with $\lim _{r \rightarrow \infty} \Theta\left(-t_{a}, r\right)=\pi / 2$ (see Fig. 7). The different solid curves for $r \geq 10$, from top to bottom, correspond to $t+t_{a}=-0.50,-0.05$, $-0.01,0.00,0.01,0.05$, and 0.50 . With increasing time, the $\Theta$ values at large $r$ move toward the constant $\Theta$ value at $r=0$. 


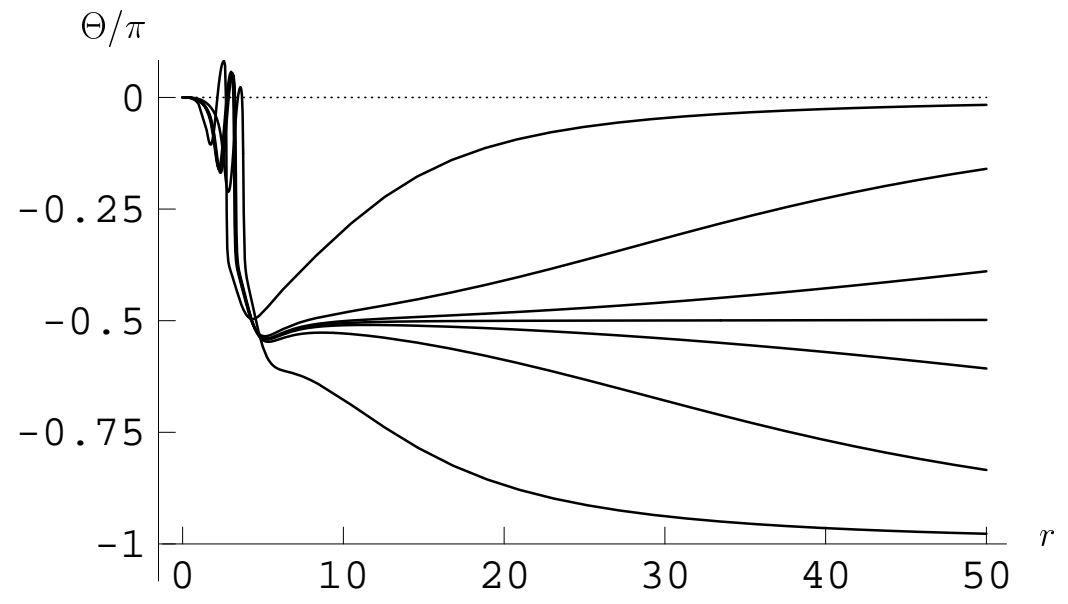

FIG. 9. Same as Fig. 8, but for times around $t=t_{a} \approx 2.924$. For $t=t_{a}$, there is a normalizable fermion zero mode, with $\lim _{r \rightarrow \infty} \Theta\left(t_{a}, r\right)=-\pi / 2$. The different solid curves for $r \geq 10$, from top to bottom, correspond to $t-t_{a}=-0.50,-0.05,-0.01,0.00,0.01,0.05$, and 0.50 . With increasing time, the $\Theta$ values at large $r$ move away from the constant $\Theta$ value at $r=0$. 